\title{
Linear and weakly nonlinear instability of a premixed curved flame under the influence of its spontaneous acoustic field
}

\author{
Raphaël C. Assier ${ }^{1, \dagger}$ and Xuesong $\mathrm{Wu}^{1}$ \\ ${ }^{1}$ Department of Mathematics, Imperial College London, South Kensington Campus, \\ London SW7 2AZ, UK \\ (Received 16 September 2013; revised 24 July 2014; accepted 4 September 2014; \\ first published online 7 October 2014)
}

The stability of premixed flames in a duct is investigated using an asymptotic formulation, which is derived from first principles and based on high-activation-energy and low-Mach-number assumptions (Wu et al., J. Fluid Mech., vol. 497, 2003, pp. 23-53). The present approach takes into account the dynamic coupling between the flame and its spontaneous acoustic field, as well as the interactions between the hydrodynamic field and the flame. The focus is on the fundamental mechanisms of combustion instability. To this end, a linear stability analysis of some steady curved flames is undertaken. These steady flames are known to be stable when the spontaneous acoustic perturbations are ignored. However, we demonstrate that they are actually unstable when the latter effect is included. In order to corroborate this result, and also to provide a relatively simple model guiding active control, we derived an extended Michelson-Sivashinsky equation, which governs the linear and weakly nonlinear evolution of a perturbed flame under the influence of its spontaneous sound. Numerical solutions to the initial-value problem confirm the linear instability result, and show how the flame evolves nonlinearly with time. They also indicate that in certain parameter regimes the spontaneous sound can induce a strong secondary subharmonic parametric instability. This behaviour is explained and justified mathematically by resorting to Floquet theory. Finally we compare our theoretical results with experimental observations, showing that our model captures some of the observed behaviour of propagating flames.

Key words: acoustics, combustion, instability

\section{Introduction}

Combustion instability, also referred to as thermo-acoustic instability, arises due to a strong interaction between the heat released by a flame and the acoustic fluctuations of a combustion chamber. When the unsteady heat release rate and acoustic fluctuations

$\dagger$ Present address: School of Mathematics, University of Manchester, Oxford Road, Manchester M13 9PL, UK. Email address for correspondence: raphael.assier@manchester.ac.uk 
are in phase, a small perturbation to the system will amplify according to the criterion of Rayleigh (1878). Combustion instability may occur in numerous real-life situations such as ramjet engines (Yu, Trouvé \& Daily 1991), rocket engines (Harrje \& Reardon 1972) and, more generally, any type of gas turbine engine (Lieuwen \& Yang 2005). The two-way interaction between the flame and the acoustics can lead to strong self-sustained fluctuations, which may have disastrous effects on the components of an engine, for example by causing vibrations and structural fatigue. It is therefore important to suppress the instability by either passive (Schadow \& Gutmark 1992) or active (Candel 2002; Dowling \& Morgans 2005) control. A significant amount of research has thus been undertaken, both theoretical (e.g. Bloxsidge, Dowling \& Langhorne 1988; Dowling 1995) and experimental (e.g. Poinsot et al. 1987; Durox et al. 2009; Steinberg et al. 2010).

Combustion is intricately multiscale in its nature, comprising a very small flame zone (where most of the thermal diffusion occurs) and an even thinner reaction sheet (where chemical reactions take place), together with the hydrodynamic and acoustic zones. The acoustic zone is comparable with the (longitudinal) size of the chamber, while the scale of hydrodynamic motion may range from the Kolmogorov length to the chamber size. Resolving such a vast range of scales presents a major challenge to direct numerical simulations of combustion in a realistic combustor. For this reason, simplified theoretical models capturing qualitatively and quantitatively the main characteristics of combustion instability are indispensable. Combustion instability is closely related not only to the intrinsic instabilities of a flame, including the Darrieus-Landau (DL) instability (Darrieus 1938; Landau 1944; Pelcé \& Clavin 1982), which is induced by the gas-expansion effect, but also to the diffusional-thermal instability, which arises primarily due to differential diffusion of heat and chemical species (Sivashinsky 1977). These instabilities are controlled by the mean heat release rate $q$ and the Lewis number $L e$, respectively. Both instabilities cause the flame to wrinkle, thereby producing unsteady heat release, which may excite acoustic modes through the thermo-acoustic effect. On the other hand, the oscillatory acoustic velocity advects the flame front, which is a kinematic effect, and furthermore the acoustic acceleration modulates the flame dynamically through the unsteady Rayleigh-Taylor (RT) effect. This mechanism was first described by Markstein (1953), Markstein \& Squire (1955) and Raushenbakh (1961), and analysed more recently by Searby \& Rochwerger (1991) and Pelcé \& Rochwerger (1992).

Combustion instability has been studied extensively using a semi-empirical approach (Ducruix et al. 2003; Lieuwen 2003), where phenomenological models are proposed for the relations between the unsteady heat release and acoustic fluctuation, bypassing detailed physical and/or chemical processes. The most direct strategy is to characterise such relations by transfer (or more generally flame-describing) functions (Ducruix, Durox \& Candel 2000; Noiray et al. 2008), and systematic experiments are then performed to extract the dependence of these functions on the frequency (and amplitude) of the sound. A somewhat less direct semi-empirical modelling is based on the so-called $G$ equation (see e.g. Markstein 1964; Kerstein, Ashurst \& Williams 1988; Dowling 1999). The latter governs kinematic advection of the flame front by the flow and acoustic velocity, and thus allows the flame surface area and hence the unsteady heat release to be calculated. Models of this kind have been further extended by Dowling (1999), Schuller, Durox \& Candel (2003) and Lieuwen (2005), and were found to give reasonably good predictions. However, such models do not take into account the dynamic effect of the acoustic acceleration on the flame. Furthermore, they ignore the so-called hydrodynamic effect, i.e. the influence of gas expansion on the ambient flow motion, and hence exclude the DL instability. 
It is worth noting that there is an important difference between a freely propagating flame and an anchored flame in their response to acoustic fluctuations. For the former, the longitudinal acoustic velocity merely causes a rigid oscillation of the flame without changing its shape or surface area, while the acoustic pressure makes a small $O(M)$ correction to the burning velocity (where $M$ is the Mach number). The mechanism of wrinkling is solely due to the dynamic response to the acoustic acceleration, which acts on the flame (i.e. a density 'discontinuity') to create the unsteady RT effect. Experimental (Searby 1992; Clanet \& Searby 1998; Clanet, Searby \& Clavin 1999; Al-Shahrany et al. 2006) and numerical (Gonzalez 1996) studies indicate that the dynamic effect of the acoustic acceleration and the DL instability are both important.

In contrast, an anchored flame may change its shape even when it is perturbed by a transversely uniform acoustic velocity. The $G$ equation approach has been applied to anchored flames to account for this kinematic effect. The dynamic RT and DL mechanisms both operate, but are ignored because of the iso-density approximation. It has been argued that the kinematic advection may be dominant for anchored flames in high-speed flows, where a strong velocity tangential to the flame advects wrinkles along the flame. Several authors (e.g. Preetham, Santosh \& Lieuwen 2008; Shin \& Lieuwen 2013) discussed extensively the validity of the iso-density approximation underpinning the $G$ equation approach, and pointed out certain applications, where the density jump across the flame is actually quite small so that the approximation may be justified. It is interesting to note that the hydrodynamic effect of density jump has been partially taken into account by the 'integral technique', which was proposed by Marble \& Candel (1979) and Yang \& Culick (1986) to study, respectively, the interaction of an acoustic wave with anchored flames and the combustion instability in a laboratory ramjet combustor. However, the approximation of averaging the velocity excludes DL instability from consideration. The DL instability has also been observed experimentally for anchored flames (see Searby \& Truffaut 2001; Searby, Truffaut \& Joulin 2001). A recent preliminary study (Luzzato et al. 2013) indicates that its effect on flame-acoustic coupling can be significant in certain cases.

The DL instability has been extensively studied using the asymptotic approach based on the large-activation-energy assumption (Matkowsky \& Sivashinsky 1979; Matalon \& Matkowsky 1982; Pelcé \& Clavin 1982), which allows the flame to be treated as a hydrodynamic discontinuity. The weakly nonlinear DL instability was investigated in the small-heat-release limit $(q \ll 1)$ by Michelson \& Sivashinsky (1977) and Sivashinsky (1977), who derived what is now referred to as the Michelson-Sivashinsky (MS) equation to describe the evolution of a flame front. Both numerical (Cambray \& Joulin 1994) and theoretical (Bychkov 1998) studies have been undertaken, emphasising the importance of DL instabilities in combustion problems. Detailed reviews of the subject can be found in Clavin $(1985,1994)$ and Bychkov \& Liberman (2000). Rigorous mathematical study of the steady states (curved and flat) of the MS equation and their linear stability has been performed by Vaynblat \& Matalon $(2000 a, b)$. A general hydrodynamic theory of flames pertaining to low-Mach-number flows was presented by Matalon \& Matkowsky (1982). As the resulting system, consisting of the Euler equations governing the flow coupled with a flame-front equation, is highly nonlinear, numerical solutions appeared only recently (Helenbrook \& Law 1999; Rastigejev \& Matalon 2006; Creta \& Matalon 2011; Altantzis et al. 2012). It has been found that a slightly perturbed flat flame develops wrinkles owing to DL instability. Its long-time nonlinear behaviour depends, inter alia, on $H$, the transverse size of the domain relative to the flame thickness. For small $H$, the flame evolves into steady cellular structures consisting of cusped crests, 
whereas for large $H$, a steady state is never reached, and instead, wrinkles develop in the region near a trough and propagate towards the crests. Application of the theory to cases with a turbulent oncoming flow indicates that DL instability modulates flame propagation substantially, and thus plays a significant role in turbulent combustion (Creta \& Matalon 2011; Fogla, Creta \& Matalon 2013). All these studies excluded acoustics from consideration since their main focus was on flame-flow interactions in open space.

In order to understand the influence of acoustic fluctuations on the dynamics of flames subject to DL instability, Markstein \& Squire (1955) first considered the stability of a flat flame in an externally imposed acoustic field, and their analysis has since been extended and refined by many authors (e.g. Searby \& Rochwerger 1991; Clanet \& Searby 1998; Bychkov 1999; Aldredge 2005). It was shown that externally imposed sound waves with a moderate amplitude may stabilise an intrinsically unstable flat flame, but at a high enough amplitude they trigger secondary parametric instabilities. However, such studies did not take into account the back-action of the flame on the acoustics, as they omitted the so-called spontaneous acoustic field, which is generated solely by the unsteady flame itself rather than being imposed externally.

Spontaneous radiation of sound waves by the flame and their impact on the flame were observed as early as the nineteenth century by Mallard \& Le Châtelier (1882) in their pioneering experiments to measure the laminar flame speed. The experiments conducted by Markstein (1953) and Searby (1992) for a flame propagating in a cylindrical tube revealed some remarkable consequences of the coupling of DL instability and acoustic modes spontaneously excited by the flame. The first (and possibly the only) mathematical investigation of the impact of spontaneous acoustic fluctuations on the stability of a curved flame was made by Pelcé \& Rochwerger (1992), who showed that changes in the flame surface area drive an exponential growth of the perturbation, as was proposed earlier by Raushenbakh (1961). However, in their study, the flame profile was modelled in an ad hoc manner by a cosine function of the transverse coordinate.

A self-consistent asymptotic theory describing acoustic-hydrodynamic-flame coupling in the flamelet regime was developed by Wu et al. (2003, hereafter WWMP) based on the work of Matalon \& Matkowsky (1982). In the low-Mach-number limit, the velocity and pressure fluctuations acquire the character of sound in the far field at large distances from the flame front, indicating that an unsteady flame spontaneously generates an acoustic field. For a flame confined in a long duct, the problem of acoustic-flame coupling is governed by an asymptotic structure consisting of four distinct regions, which describe the acoustics, the hydrodynamics, heat transfer and chemical reaction, and more importantly the intricate interplay among them. The resulting interactive system consists of the Euler equations (which govern the hydrodynamics) coupled to the acoustic equations, along with an equation governing the flame front. In this theory, the nature of the acoustic-flame interaction is brought to light explicitly: flame wrinkling modulates its surface area and hence the heat release to drive acoustic modes of the chamber, and the acceleration associated with the sound wave in turn creates an unsteady RT effect, by which the sound wave exerts a back-effect on the hydrodynamics and therefore on the flame. Thus, in a confined domain, DL instability and acoustic fluctuations are intrinsically coupled, and the instability of a premixed combustion is most likely to be different from the situations where acoustics is (artificially) excluded; the latter case is of relevance only for flames in unbounded domains, or when a flame ultimately evolves into a steady state. The mathematical formulation of WWMP has been adapted in Wu \& 
Law (2009) to investigate the interactions of a flat flame with weak turbulence in the oncoming mixture. The theory was further generalised by $\mathrm{Wu} \&$ Moin (2010) to account for the influence of enthalpy fluctuations in the oncoming mixture on flame-flow-acoustic interactions. In the applications considered in Wu et al. (2003), Wu \& Law (2009) and Wu \& Moin (2010), the steady state is that of a flat flame, for which the acoustic-flame coupling term is nonlinear: the acoustic source involves the quadratic product of the gradient of the perturbed flame front, while the back-action of the sound is represented by the product of the acoustic acceleration with the flame front.

In the present paper, we apply the formalism of WWMP to steady curved flames. In this case, a linear perturbation to the flame front necessarily induces a linear acoustic disturbance, which simultaneously acts on the flame through a linear coupling term. Our first and primary aim is to assess how the coupling changes the stability properties predicted by ignoring the spontaneous acoustic perturbation. Our second aim is to propose an improved model, which accounts for both the spontaneous acoustics and DL instability. Such a model could be useful in designing and testing active controllers to suppress the instability (Dowling \& Morgans 2005). In the present study, the model will be used to predict some key experimental observations made by Searby (1992), especially the subharmonic parametric instability, which remains poorly understood.

The rest of the paper is structured as follows. In $\S 2$, the problem is formulated, and the asymptotic description of the flame-flow-acoustic interaction is explained briefly. The composite theory of second-order accuracy, formulated in Wu \& Law (2009), is summarised in $\S 2.1$ to serve as a starting point for the present investigation. In particular, we point out in $\S 2.2$ that a perturbation to a curved flame would always generate a spontaneous acoustic perturbation, and that the two are coupled in a linear manner. In order to make analytical progress, in $\S 2.3$ we make a simplifying assumption of weak nonlinearity, which allows us to linearise the hydrodynamic equations and jump conditions while retaining the geometric nonlinearity in the front equation. This simplification, though not entirely justifiable by a systematic asymptotic analysis, renders the problem analytically and computationally tractable, leading to a relatively simple model capable of qualitatively predicting some key experimental observations. The steady states of the simplified system are equivalent to those of the MS equation when gravity is absent. An important parameter, $\gamma$, inversely proportional to the flame thickness, will be introduced to classify the steady solutions. These steady flames are known to be stable (see Vaynblat \& Matalon 2000a,b) when the spontaneous acoustic perturbations are ignored. In $\S 3$, we perform a linear stability analysis including the spontaneous acoustic perturbations, and demonstrate that these steady flames are actually linearly unstable. In order to corroborate these results and also to study the influence of nonlinearity, we derive in $\S 4$ the evolution equations governing the linear and weakly nonlinear development of the perturbed flame coupled with the spontaneously generated acoustic field. Numerical methods are developed to solve the coupled system. In $\S 5$ we show that numerical solutions of the initial-value problem not only confirm the linear instability of the steady curved flames, but also describe how the flame evolves nonlinearly with time. In particular, the results predict that the spontaneous sound of the flame induces a secondary parametric instability as observed in the experiments of Searby (1992). The onset of this instability is justified mathematically by resorting to Floquet theory. Finally, in $\S 6$, we summarise our results and briefly discuss their implications and possible extensions. The mathematical formulation will first be presented for a full three-dimensional rectangular duct, but will, for simplicity, be specialised to the two-dimensional case from $\S 3$, for which numerical computations will be carried out. 


\section{Formulation and asymptotic description of the problem}

We consider premixed combustion in a long duct with a width $h^{*}$ and length $\ell^{*} \gg h^{*}$. The fresh mixture enters the duct at a constant mean velocity $U_{-}^{*}$, and has a mean density $\rho_{-\infty}$ and temperature $\Theta_{-\infty}$. We assume for simplicity that the combustion takes place through a one-step irreversible chemical reaction, and that the mixture, consisting of a single deficient reactant and an abundant component, is Newtonian and obeys the state equation for a perfect gas. The temperature rises to $\Theta_{\infty}$ behind the flame. A key parameter describing the reaction is the Zeldovich number,

$$
\beta=\frac{E\left(\Theta_{\infty}-\Theta_{-\infty}\right)}{\mathscr{R} \Theta_{\infty}^{2}},
$$

where $E$ is the dimensional activation energy and $\mathscr{R}$ is the universal gas constant. The flame is characterised by a laminar flame speed $U_{L}$, at which the flame propagates into the fresh mixture, and an intrinsic thickness $d=D_{t h}^{*} / U_{L}$, where $D_{t h}^{*}$ is the thermal conductivity, which is, along with the viscosity and mass diffusivity, determined by the abundant component of the mixture. The reference length, time, velocity, density and temperature are taken to be $h^{*} /(2 \pi), h^{*} /\left(2 \pi U_{L}\right), U_{L}, \rho_{-\infty}$ and $\Theta_{-\infty}$, respectively. The resulting non-dimensional space, time, velocity, density and temperature variables are denoted by $(x, y, z), t, \boldsymbol{u} \equiv(u, v, w), \rho$ and $\theta$. The non-dimensional pressure $p$ is defined by writing the dimensional pressure as $\left(p_{-\infty}+\rho_{-\infty} U_{L}^{2} p\right)$, where $p_{-\infty}$ is the atmospheric pressure. The velocity, pressure, temperature and fuel mass fraction $Y$ satisfy the non-dimensional Navier-Stokes (NS) equations for reactive flows, with the reaction rate $\Omega$ being described by the Arrhenius law,

$$
\Omega=\Omega_{0} \rho Y \exp \left\{\beta\left(\frac{1}{\Theta_{+}}-\frac{1}{\theta}\right)\right\},
$$

where $\Theta_{+}=1+q$ is the adiabatic flame temperature, with $q$ being the mean heat release rate, and $\Omega_{0}$ is a constant, chosen such that the non-dimensional speed of a flat flame is unity. In the resulting equations, representing conservation of mass, momentum and energy, transport of the species and the state of the mixture, the following parameters appear: the Prandtl number $P r$, the Lewis number $L e$, the activation energy $\beta$, the normalised gravitational force $G=g h^{*} /\left(2 \pi U_{L}^{2}\right)$ in the $x$ direction, as well as the aspect ratio $\delta$ and the Mach number $M$, which are defined as

$$
\delta=2 \pi d / h^{*}, \quad M=U_{L} / a^{*},
$$

where $a^{*}$ is the speed of sound.

Asymptotic theories for combustion have been developed by assuming a large activation energy, $\beta \gg 1$, plus the requirement that the Lewis number $L e$ is close to unity, or more precisely

$$
L e=1+\beta^{-1} l \quad \text { with } l=O(1) .
$$

The reaction takes place in a thin region with a width of $O(\delta / \beta)$. On the scale of $\delta$, the flame front appears as an interface separating the burnt and unburnt materials, and can be represented mathematically by $x=f(y, z, t)$, as illustrated in figure 1 . It is convenient to formulate the problem in the flame frame of reference $(\xi, \eta, \zeta, \tau)$ by introducing

$$
\xi=x-f(y, z, t), \quad \eta=y, \quad \zeta=z \quad \text { and } \quad \tau=t,
$$




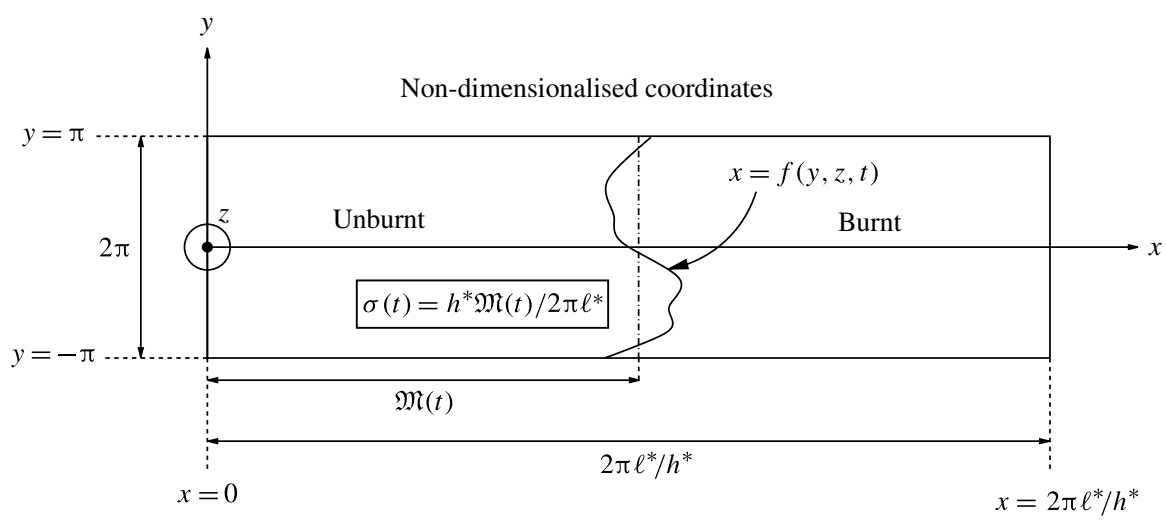

FIgURE 1. A diagrammatic illustration of the problem and the definition of the mean flame position $\mathfrak{M}$ (dashed line) and $\sigma$ (normalised).

and writing the velocity field as $\boldsymbol{u}=u \boldsymbol{e}_{\xi}+\boldsymbol{v}$, where $\boldsymbol{e}_{\xi}$ is the unit vector in the $\xi$ direction. The reader is reminded that the explicit parametrisation $x=f(y, z, t)$ of the flame is possible only before flame folding takes place; if the latter occurs, the formulation must be recast in terms of a level set function, $\phi(x, y, z, t)$, introduced such that the flame front is specified as the contour $\phi(x, y, z, t)=0$, and at an arbitrary point $(x, y, z), \phi$ represents the distance of this point from the flame (see e.g. Williams 1985).

The hydrodynamic theory of flames (Matalon \& Matkowsky 1982; Pelcé \& Clavin 1982) was formulated in the so-called flamelet regime and for low-Mach-number flows, which correspond to the assumptions that

$$
\delta \ll 1 \quad \text { and } \quad M \ll 1 .
$$

The flame-flow interaction involves three asymptotic regions (figure 2), i.e. the reaction, preheat and hydrodynamic zones corresponding to $\xi=O(\delta / \beta), O(\delta)$ and $O(1)$, respectively. The reaction and preheat zones constitute the inner structure of the flame. Through the gas expansion associated with heat release, the flame impacts the fluid motion in an $O(1)$ region on each side of the flame. The motion on each side is incompressible to leading order, but the density $R$ takes different constant values, namely

$$
R= \begin{cases}R_{+}=(1+q)^{-1} & \text { if } \xi>0, \\ R_{-}=1 & \text { if } \xi<0,\end{cases}
$$

while the temperature, to leading order, is defined by $\Theta=1 / R$. The solution for the flow field and flame front expands as

$$
(u, \boldsymbol{v}, p, f)=\left(u_{0}, \boldsymbol{v}_{0}, p_{0}, f_{0}\right)+\delta\left(u_{1}, \boldsymbol{v}_{1}, p_{1}, f_{1}\right)+\cdots .
$$

The leading-order flow field $\left(u_{0}, \boldsymbol{v}_{0}, p_{0}\right)$ is governed by the Euler equations, and the $O(\delta)$ correction satisfies the linearised Euler equations with the viscous correction appearing as inhomogeneous terms. Across the flame front $\xi=0$, there exist jumps in the leading-order velocity and pressure as well as in their $O(\delta)$ corrections. One may 


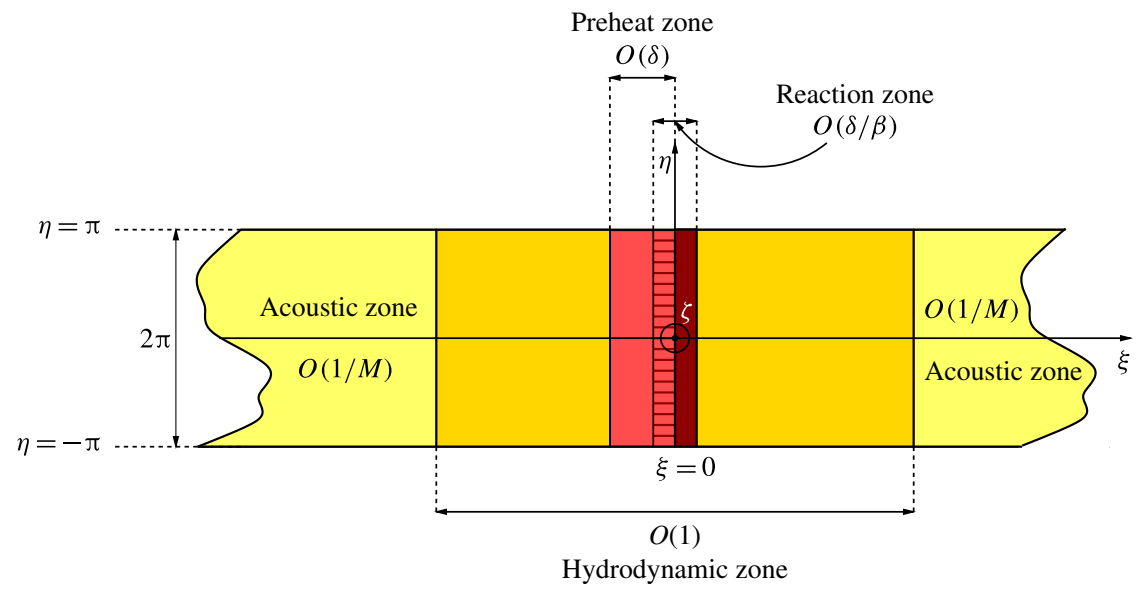

FIGURE 2. (Colour online) Different asymptotic zones.

combine the first two terms to formulate a composite approximation accurate up to $O(\delta)$, in which case $u$ and $v$ satisfy the NS equations and the jump conditions

$$
\begin{aligned}
& {[u]_{-}^{+}=q\left[1+(\tilde{\nabla} f)^{2}\right]^{-1 / 2}+\delta J_{u},} \\
& {[\boldsymbol{v}]_{-}^{+}=-q \tilde{\nabla} f\left[1+(\tilde{\nabla} f)^{2}\right]^{-1 / 2}+\delta \boldsymbol{J}_{v},} \\
& {[p]_{-}^{+}=-q+\delta J_{p},}
\end{aligned}
$$

where the gradient operator $\tilde{\nabla}=(\partial / \partial \eta, \partial / \partial \zeta)^{\mathrm{T}}$, and []$_{-}^{+}$denotes a jump across the flame front, defined such that, for any function $\phi(\xi, \eta, \zeta, \tau)$,

$$
[\phi]_{-}^{+}=\phi\left(0^{+}, \eta, \zeta, \tau\right)-\phi\left(0^{-}, \eta, \zeta, \tau\right) .
$$

The $O(\delta)$ terms in the jumps were first derived independently by Matalon \& Matkowsky (1982), and by Pelcé \& Clavin (1982) in the linear limit. Their expressions have not been written explicitly for brevity but can be found in Wu $\&$ Law (2009). The Euler equations are coupled with a flame-front equation (Matalon \& Matkowsky 1982)

$$
\frac{\partial f}{\partial \tau}=u\left(0^{-}, \eta, \zeta, \tau\right)-\boldsymbol{v}\left(0^{-}, \eta, \zeta, \tau\right) \cdot \tilde{\nabla} f-\left[1+(\tilde{\nabla} f)^{2}\right]^{1 / 2}+\delta M_{a}\left(\tilde{\nabla}^{2} f+\Gamma_{s}\right),
$$

where the expression for $\Gamma_{s}$ can be found in Matalon \& Matkowsky (1982) and $\mathrm{Wu}$ $\&$ Law (2009). The Markstein number $M_{a}$ is given by

$$
M_{a}=\frac{1+q}{q} \ln (1+q)+\frac{l}{2} \int_{0}^{\infty} \ln \left(1+q \mathrm{e}^{-x}\right) \mathrm{d} x
$$

for a one-step irreversible Arrhenius reaction (Clavin \& Williams 1982). Note that the normal burning velocity is defined with respect to the fresh mixture just upstream of the flame. As discussed in Clavin \& Williams (1982), and in more depth in Clavin \& Graña Otero (2011), a normal burning velocity may alternatively be defined in the burnt mixture just downstream of the flame, in which case a different expression for $M_{a}$ would appear. 


\subsection{Flame-flow-acoustic interaction theory of Wu et al. (2003)}

It was shown in WWMP that the longitudinal velocity $u$ exhibits a jump across the hydrodynamic zone, implying that an unsteady flame would generate a spontaneous sound. In a long duct, an outer acoustic region corresponding to $\xi=O(1 / M)$ arises. The spontaneous sound also acts on the flame. The flame-flow-acoustic coupling is described by four asymptotic regions. With the preheat and reaction zones being treated analytically, the direct flame-acoustic interaction is between the acoustic and hydrodynamic regions.

\subsubsection{The acoustic zone}

In view of its longitudinal size, the acoustic field is described by the stretched variable $\tilde{\xi}=M \xi$. The flow quantities are expanded in terms of $M$. Among them, the acoustic velocity $u_{a}$ and pressure $p_{a}$ are introduced by writing

$$
u=U_{ \pm}+u_{a}(\tilde{\xi}, \tau)+O(M), \quad p=M^{-1}\left\{p_{a}(\tilde{\xi}, \tau)-R G \tilde{\xi}\right\}+O(1)
$$

and they are found to satisfy the linear equations

$$
\frac{\partial p_{a}}{\partial \tau}+\frac{\partial u_{a}}{\partial \tilde{\xi}}=0, \quad R \frac{\partial u_{a}}{\partial \tau}+\frac{\partial p_{a}}{\partial \tilde{\xi}}=0 .
$$

Across the hydrodynamic zone, the pressure $p_{a}$ is continuous, and the velocity $u_{a}$ exhibits a jump, that is,

$$
\llbracket p_{a} \rrbracket_{-}^{+}=0, \quad \llbracket u_{a} \rrbracket_{-}^{+}=\mathscr{J}_{a}(\tau)=q\left\{\overline{\left[1+(\tilde{\nabla} f)^{2}\right]^{1 / 2}}-1\right\}+O(\delta),
$$

where $\llbracket \rrbracket_{-}^{+}$represents the jump across the hydrodynamic zone, defined such that, for any function $\phi(\tilde{\xi}, \tau)$, we have $\llbracket \phi \rrbracket_{-}^{+}=\phi\left(0^{+}, \tau\right)-\phi\left(0^{-}, \tau\right)$, and the overbar denotes a space average in the $\eta-\zeta$ plane. The leading-order part of (2.17) indicates that the acoustic velocity jump is, to leading order, proportional to the surface area of the flame front, a fact that has been known and used since Markstein (1970). The $O(\delta)$ terms of (2.17) were given in Wu \& Law (2009).

\subsubsection{The hydrodynamic zone}

In order to facilitate the matching with the acoustic field, the solution for the velocity, pressure and flame front is decomposed as

$$
\left.\begin{array}{l}
p=M^{-1} p_{a}(0, \tau)+P_{ \pm}+\left(\frac{\partial p_{a}}{\partial \tilde{\xi}}\left(0^{ \pm}, \tau\right)-R G\right)(F+\xi)+P \\
u=U_{ \pm}+u_{a}\left(0^{ \pm}, \tau\right)+U, \quad v=V, \quad f=F_{a}+F
\end{array}\right\}
$$

where $F_{a}$ is chosen such that

$$
F_{a}^{\prime}(t)=U_{-}-1+u_{a}\left(0^{-}, t\right)
$$

with which the flame-front equation (2.13) becomes

$$
\frac{\partial F}{\partial \tau}=U\left(0^{-}, \eta, \zeta\right)-\boldsymbol{V}\left(0^{-}, \eta, \zeta\right) \cdot \tilde{\nabla} F-\left\{\left[1+(\tilde{\nabla} F)^{2}\right]^{1 / 2}-1\right\}+\delta M_{a} \tilde{\nabla}^{2} F
$$


Here we have retained only the $\tilde{\nabla}^{2} F$ term, which is important, as it provides a large cutoff wavenumber to render the initial-value problem well posed. Other terms of $O(\delta)$ can be included as was done in Wu \& Law (2009). Those terms were found to make a small quantitative modification, and are expected to behave similarly in the present problem. They are neglected since our aim at this stage is to propose a relatively simple model, and use it to predict the qualitative behaviour of the flame and the spontaneous sound.

Using (2.18), one obtains the equations governing the hydrodynamic zone:

$$
\left.\begin{array}{rl}
\frac{\partial U}{\partial \xi}+\tilde{\nabla} \cdot \boldsymbol{V} & =\frac{\partial \boldsymbol{V}}{\partial \xi} \cdot \tilde{\nabla} F, \\
R\left\{\frac{\partial U}{\partial \tau}+S \frac{\partial U}{\partial \xi}+\boldsymbol{V} \cdot \tilde{\nabla} U\right\}+\frac{\partial U}{\partial \xi} & =-\frac{\partial P}{\partial \xi}+\delta \operatorname{Pr} \tilde{\Delta} U, \\
R\left\{\frac{\partial \boldsymbol{V}}{\partial t}+S \frac{\partial \boldsymbol{V}}{\partial \xi}+\boldsymbol{V} \cdot \tilde{\nabla} \boldsymbol{V}\right\}+\frac{\partial \boldsymbol{V}}{\partial \xi} & =-\tilde{\nabla} P+\tilde{\nabla} F \frac{\partial P}{\partial \xi}+\delta \operatorname{Pr} \tilde{\Delta} \boldsymbol{V} .
\end{array}\right\}
$$

Here, using $\mathbb{H}$ to denote the usual Heaviside function, we have put

$$
S=U-F_{\tau}-\boldsymbol{V} \cdot \tilde{\nabla} F+\mathscr{J}_{a}(\tau) \mathbb{H}(\xi) .
$$

Owing to the definition of $F$, we have $\tilde{\nabla} f=\tilde{\nabla} F$, and hence

$$
\mathscr{J}_{a}(\tau)=q\left\{\overline{\left[1+(\tilde{\nabla} F)^{2}\right]^{1 / 2}}-1\right\}+O(\delta) .
$$

Across the flame front, the longitudinal and transverse velocities and the pressure satisfy the jump conditions

$$
\left.\begin{array}{l}
{[U]_{-}^{+}=q\left[1+(\tilde{\nabla} F)^{2}\right]^{-1 / 2}-q-\mathscr{J}_{a}(\tau)+\delta J_{U},} \\
{[\boldsymbol{V}]_{-}^{+}=-q(\tilde{\nabla} F)\left[1+(\tilde{\nabla} F)^{2}\right]^{-1 / 2}+\delta J_{V},} \\
{[P]_{-}^{+}=-\left(\mathscr{B}_{a}(\tau)+\frac{q G}{1+q}\right) F+\delta J_{P},}
\end{array}\right\}
$$

where the expressions for the $O(\delta)$ terms in (2.24) as well as the definition of the Laplace operator $\tilde{\Delta}$ in (2.21) are given in Wu \& Law (2009), but are omitted here for brevity. The function $\mathscr{B}_{a}(\tau)$ represents the jump of $\partial p_{a} / \partial \tilde{\xi}$ across the hydrodynamic zone:

$$
\mathscr{B}_{a}(\tau)=\llbracket \frac{\partial p_{a}}{\partial \tilde{\xi}} \rrbracket_{-}^{+}=\llbracket-R \frac{\partial u_{a}}{\partial \tau} \rrbracket_{-}^{+} .
$$

The system describing flame-flow-acoustic interactions consists of (2.20) and (2.21), which are coupled to (2.16) via (2.23) and (2.24). The flame-acoustic coupling is represented by $\mathscr{J}_{a}$ and $\mathscr{B}_{a}$. Through $\mathscr{J}_{a}$, the flame excites acoustic fluctuations, which in turn act on the flame through the unsteady RT effect created by the acoustic acceleration $\mathscr{B}_{a}$. Note that the acoustic velocity $u_{a}\left(0^{-}, t\right)$ drops out of the system, and thus kinematic advection by the acoustic velocity plays no role in the dynamics of a freely propagating flame. 


\subsection{Stability of a curved flame}

The analysis in the previous subsection shows that an unsteady flame in general produces an acoustic field, which is absent only when the flame is steady. Such steady states are relevant only if they are stable. In this subsection, the general formulation is used to study the linear instability of a steady curved flame. Let the steady hydrodynamic field and the flame front be denoted by $\left(U^{S}, V^{S}, P^{S}, F^{S}\right)$. They satisfy the steady version of (2.20) and (2.21), that is, the time derivative $\partial / \partial \tau$ in (2.21) is dropped, and $\partial F^{S} / \partial \tau=U_{F}$ in (2.20), where $U_{F}$ is the steady propagation velocity of the flame. The perturbed flow and flame front can be written as

$$
(U, \boldsymbol{V}, P, F)=\left(U^{S}, \boldsymbol{V}^{S}, P^{S}, F^{S}\right)+(\tilde{u}, \tilde{\boldsymbol{v}}, \tilde{p}, \tilde{F}) .
$$

Substituting (2.26) into (2.20), (2.21) and (2.24), and linearising, we obtain the equations governing the perturbation, as well as the corresponding jump conditions, which for brevity, we decide not to write out. For a curved flame, a uniform mean flow

$$
u_{a}^{S}=q\left\{\overline{\left[1+\left(\tilde{\nabla} F^{S}\right)^{2}\right]^{1 / 2}}-1\right\} \quad \text { for } \xi>0,
$$

is generated in the outer acoustic region downstream of the flame. The perturbed field in the acoustic zone can be written as

$$
\left(u_{a}, p_{a}\right)=\left(u_{a}^{S}, 0\right)+\left(\tilde{u}_{a}, \tilde{p}_{a}\right) .
$$

Substitution into (2.16) shows that $\tilde{u}_{a}$ and $\tilde{p}_{a}$ remain governed by

$$
\frac{\partial \tilde{p}_{a}}{\partial \tau}+\frac{\partial \tilde{u}_{a}}{\partial \tilde{\xi}}=0, \quad R \frac{\partial \tilde{u}_{a}}{\partial \tau}+\frac{\partial \tilde{p}_{a}}{\partial \tilde{\xi}}=0 .
$$

It follows from (2.17) that the linearised jump condition is

$$
\llbracket \tilde{u}_{a} \rrbracket_{-}^{+}=\tilde{\mathscr{J}}_{a}(\tau)=q \overline{\nabla F^{S} \cdot \nabla \tilde{F}\left[1+\left(\tilde{\nabla} F^{S}\right)^{2}\right]^{-1 / 2}} .
$$

The above relation indicates that a perturbation to a curved flame must generate spontaneously an acoustic fluctuation of the same order of magnitude. This is very different from the case of a steady flat flame, where the spontaneous sound arises at the quadratic order of the flame-front perturbation. A correct formulation for the stability of a curved flame must therefore take into account the acoustic perturbation, which may fundamentally change the stability behaviour, as will be shown in $\S 3$.

\subsection{Simplified flame-flow-acoustic interaction model with linear hydrodynamics}

The general flame-flow-acoustic interaction system in $\S 2.1$ and the instability problem formulated in $\S 2.2$ represent a formidable computational challenge. The main obstacle lies in the hydrodynamics of the steady state and the perturbation. In order to make analytical progress, we shall assume that the hydrodynamic field as well as the gradient of the flame are small so that the equations (2.21) can be linearised, leading to a reduced system for the hydrodynamics,

$$
\frac{\partial U}{\partial \xi}+\tilde{\nabla} \cdot \boldsymbol{V}=0, \quad R \frac{\partial U}{\partial \tau}+\frac{\partial U}{\partial \xi}=-\frac{\partial P}{\partial \xi}, \quad R \frac{\partial \boldsymbol{V}}{\partial \tau}+\frac{\partial \boldsymbol{V}}{\partial \xi}=-\tilde{\nabla} P .
$$


The jump conditions (2.24) can also be linearised to give

$$
[U]_{-}^{+}=0, \quad[V]_{-}^{+}=-q \tilde{\nabla} F, \quad[P]_{-}^{+}=-\left[\mathscr{B}_{a}(\tau)+q G /(1+q)\right] F .
$$

The geometric nonlinearity in the front equation (2.20) presents no substantial difficulty and could be retained in full, but, in order to connect the present model with the well-known MS equation, we write, on the assumption of weak nonlinearity, $\left(1+(\tilde{\nabla} F)^{2}\right)^{1 / 2} \approx 1+(\tilde{\nabla} F)^{2} / 2$, and so the flame equation (2.20) simplifies to

$$
\frac{\partial F}{\partial \tau}=U\left(0^{-}, \eta, \zeta\right)-\frac{1}{2}(\tilde{\nabla} F)^{2}+\delta M_{a} \tilde{\nabla}^{2} F .
$$

The reason for retaining the geometric nonlinearity is that a curved steady flame may form despite linear hydrodynamics.

The equations governing the acoustics remains intact, namely,

$$
\frac{\partial p_{a}}{\partial \tau}+\frac{\partial u_{a}}{\partial \tilde{\xi}}=0, \quad R \frac{\partial u_{a}}{\partial \tau}+\frac{\partial p_{a}}{\partial \tilde{\xi}}=0,
$$

but the jump conditions are simplified to

$$
\llbracket p_{a} \rrbracket_{-}^{+}=0, \quad \llbracket u_{a} \rrbracket_{-}^{+}=\mathscr{J}_{a}(\tau)=\frac{q}{2} \overline{(\tilde{\nabla} F)^{2}} .
$$

In order to impose boundary conditions at the extremities of the duct, it is necessary to define the mean position of the flame $\mathfrak{M}(t)$ and the normalised mean position of the flame $\sigma(t)$. For the two-dimensional case, we can write

$$
\sigma(t)=\frac{h^{*}}{2 \pi \ell^{*}} \mathfrak{M}(t) \quad \text { with } \mathfrak{M}(t)=\frac{1}{2 \pi} \int_{-\pi}^{\pi} f(y, t) \mathrm{d} y .
$$

Figure 1 illustrates the definition of $\mathfrak{M}$. Since we have $\xi=x-f(y, t)$, integrating this with respect to $y$ between $-\pi$ and $\pi$, one obtains $\xi=x-\mathfrak{M}(t)$. Setting $x=$ 0 leads to $\tilde{\xi}=-\sigma L$, while setting $x=2 \pi \ell^{*} / h^{*}$ gives $\tilde{\xi}=(1-\sigma) L$, where $L=$ $2 \pi M \ell^{*} / h^{*}$. Consequently, the boundary conditions can be specified. For a duct with a closed(open) end at the left(right)-hand side, the following conditions apply:

$$
u_{a}(-\sigma L, \tau)=0 \quad \text { and } \quad p_{a}((1-\sigma) L, \tau)=0 .
$$

As is illustrated in figure 2, the position where the boundary conditions are applied may differ slightly from the exact boundary of the domain. However, since the difference is much smaller than $O(1 / M)$, it does not influence the acoustics to leading-order accuracy.

In the rest of this paper, we shall focus our attention on the system formed by the hydrodynamic equation (2.31) and jump conditions (2.32), the weakly nonlinear flame-front equation (2.33) and the acoustic equation (2.35). The approximation leading to this system, namely linearising the hydrodynamic while retaining the geometric nonlinearity, requires further explanations. In the absence of an acoustic field, the approximation can be justified asymptotically in the limit of small heat release (Sivashinsky 1977). Interestingly, the conclusion holds also for the present case, as the asymptotic analysis can be generalised to derive, in a consistent fashion, a reduced system governing the interaction and evolution of the flame and the 
spontaneous sound. A key observation in the analysis is that the forcing from the flame wrinkling, though of small amplitude $\left(O\left(q^{3}\right)\right)$, can generate a much stronger acoustic wave (eigenmode) because it is in resonance with the latter; the details will be given in a future paper. For $O(1)$ heat release, the approximation is no longer self-consistent and becomes a purely tactical one for deriving a relatively simple model, and so the system (2.31)-(2.35) may be referred to as the flame-flow-acoustic interaction model. Its validity is necessarily restricted to situations where the flow motion is relatively weak. In typical laboratory experiments (e.g. Markstein 1953; Searby 1992), the hydrodynamic motion does not appear to be vigorous despite the heat release being of $O(1)$. The simplified system has the merit of being mathematically tractable, while on the other hand it retains all relevant physical factors causing combustion instability, including advection of the flame by the flow, geometric nonlinearity, the full hydrodynamic instability, the generation of spontaneous sound and its back-action on the flame via the unsteady RT effect. With further assumptions, the system may be reduced to even simpler flame models such as the $G$ equation and the MS equation.

\section{Linear stability analysis}

\subsection{Steady flames and their stability with the spontaneous acoustics excluded}

As shown in appendix A, for steady two-dimensional flames, the system (2.31)-(2.33) is reduced to (A 6). When gravity is absent $(G=0)$, (A 6) is, as expected, equivalent to the well-known MS equation

$$
\left.\begin{array}{l}
\frac{\partial \varphi}{\partial t}=\frac{1}{2} I(\varphi ; \eta)+\frac{1}{\gamma} \frac{\partial^{2} \varphi}{\partial \eta^{2}}+\frac{1}{2}\left(\frac{\partial \varphi}{\partial \eta}\right)^{2}, \\
I(\varphi ; \eta)(k, t)=|k| \widehat{\varphi}(k, t),
\end{array}\right\}
$$

where the operator $I(\varphi, \eta)=\mathscr{H}(\partial \varphi / \partial \eta, \eta)$, with $\mathscr{H}$ being the Hilbert transform. The variables and parameters in (A 6) and (3.1) are simply related via $t=q \tau, \varphi=-F / q$ and

$$
\delta M_{a}=q / \gamma
$$

Vaynblat \& Matalon (2000a) presented some rigorous results concerning the MS equation, which we summarise briefly. The MS equation admits $\mathfrak{m}$-pole solutions of the form

$$
\varphi_{m}(\eta, t)=c_{0}(t)+\frac{2}{\gamma} \sum_{m=1}^{\mathfrak{m}} \ln \left[\frac{1}{2}\left\{\cosh \left(y_{m}(t)\right)-\cos \left(\eta-x_{m}(t)\right)\right\}\right],
$$

with $\mathfrak{m}$ pairs of complex conjugate poles $z_{m}(t)=x_{m}(t)+\mathrm{i} z_{m}(t)$. The maximum number of poles that may exist depends on $\gamma$ and is given by $\mathfrak{m}_{\max }=\operatorname{Int}[\gamma / 2]$. A coalescent pole solution is a pole solution such that the poles are aligned vertically. A steady coalescent pole solution is a coalescent pole solution such that the location of the poles is time-independent. Vaynblat \& Matalon (2000a) showed that, for a given pair $(\gamma, \mathfrak{m})$, there exists a steady coalescent pole solution only if $\mathfrak{m} \leqslant \mathfrak{m}_{0}(\gamma)$, where $\mathfrak{m}_{0}(\gamma)=\operatorname{Int}[\gamma / 4+1 / 2]$. In addition, for a given $\mathfrak{m} \leqslant \mathfrak{m}_{0}(\gamma)$, this solution is unique and is denoted $\varphi_{\mathfrak{m}}(\gamma, \eta, t)$. The steady coalescent one- and two-pole solutions are conveniently used to verify our numerical code and solutions. The value chosen for the parameter $\gamma$ (related to $\delta M_{a}$ ) should affect the type of steady states obtained. In our numerical approach, we only compute the Fourier coefficients for $n \neq 0$, which suffices since our aim is to model the shape of a flame moving freely in the duct. 
(a)

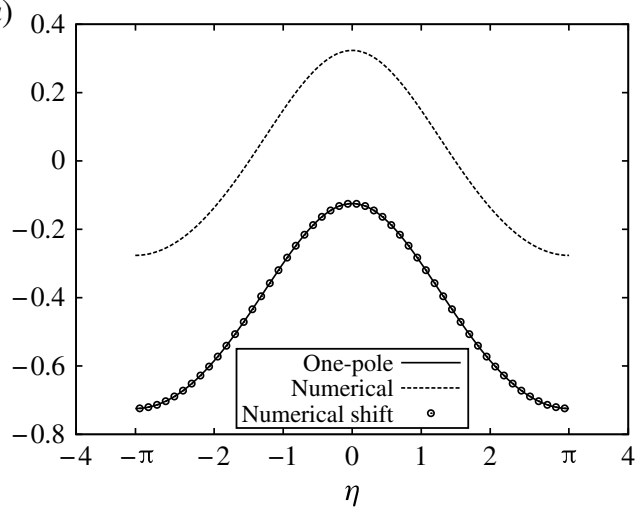

(b)

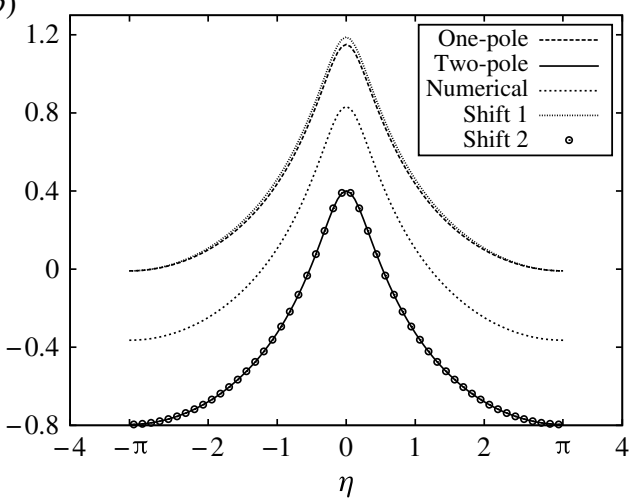

FIGURE 3. Comparison of the analytical steady solutions $\left(-\varphi_{1,2}\right)$ of the form (3.3) and the numerical steady solutions $(F / q)$ for $(a) \gamma=2.1$ and $(b) \gamma=6.2$.

The coefficient $\widehat{F}_{0}$ describes the translation along the duct, and so does the function $c_{0}(t)$ in (3.3). Hence equivalent steady states computed with the two methods may differ by a constant. The comparison between our numerical solutions and analytical ones is shown in figure 3 for $M=0.0007$ (corresponding to a laminar flame speed $\left.U_{L}=0.24 \mathrm{~m} \mathrm{~s}^{-1}\right), q=5.25$ and $N=30$. From figure 3 , it is clear that, in the case of $\gamma=2.1 \quad\left(\mathfrak{m}_{0}=1\right)$, we have found numerically the unique one-pole steady solution, while for $\gamma=6.2\left(\mathfrak{m}_{0}=2\right)$, we have found the unique two-pole solution.

The stability of all possible steady solutions of the MS equation has been investigated rigorously in Vaynblat \& Matalon $(2000 a)$. For a steady solution $\varphi_{\mathfrak{m}}$, the evolution of a small perturbation $\psi$ is studied by writing $\varphi=\varphi_{\mathfrak{m}}+\psi$. Linearising the MS equation (3.1) about $\varphi_{\mathfrak{m}}$ leads to the equation for $\psi$,

$$
\left.\begin{array}{l}
\frac{\partial \psi}{\partial t}=\frac{1}{2} I(\psi ; \eta)+\frac{1}{\gamma} \frac{\partial^{2} \psi}{\partial \eta^{2}}+\frac{\partial \varphi_{\mathfrak{m}}}{\partial \eta} \frac{\partial \psi}{\partial \eta}, \\
\widehat{I\{\psi ; \eta}\}(k, t)=|k| \widehat{\psi}(k, t) .
\end{array}\right\}
$$

The results of Vaynblat \& Matalon (2000a) are summarised in figure 4. It has been found that, for each value of $\gamma$, only one of the known steady solutions is stable and that this solution is always the one with the maximum number of poles allowed. In our case, we have $\gamma_{1}=2, \gamma_{2}=6$ and $\gamma_{3}=10$. In particular, we have $\gamma_{1}<2.1<\gamma_{2}$ and $\gamma_{2}<6.2<\gamma_{3}$. Hence the steady solutions of figure 3 captured numerically are found to be linearly stable in Vaynblat \& Matalon (2000a) within the system (3.4). However, their stability analysis was performed by assuming that the perturbed flame front remains governed by the MS equation (3.1). The latter was derived by neglecting two main quantities: the time derivative of the perturbed hydrodynamic field and the spontaneous acoustic fluctuations. In this section we aim to study the linear stability of the steady solutions proved to be stable in Vaynblat \& Matalon (2000a), taking full account of both the spontaneous acoustic perturbations and the unsteadiness of the hydrodynamic field.

\subsection{Instability including the spontaneous acoustics}

Instead of the MS equation (3.1), the starting point of the present stability analysis is the interactive system (2.31)-(2.33) and (2.34)-(2.35). The perturbed acoustics, flame 


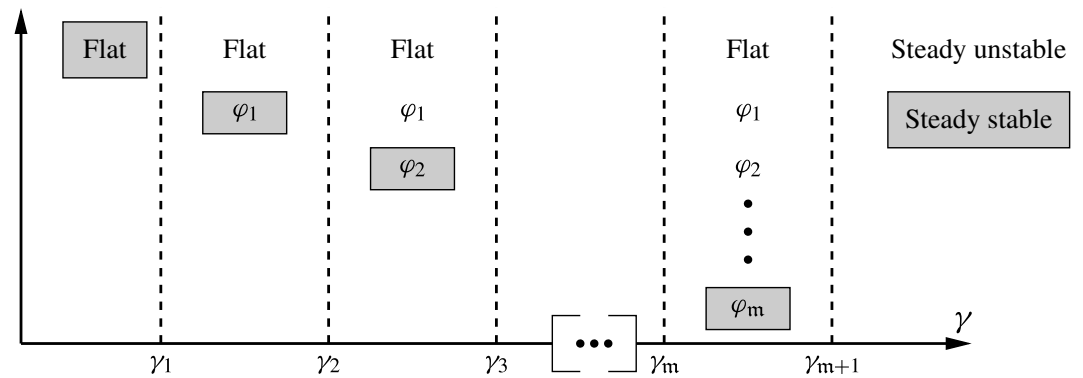

FIGURE 4. Summary of stability results concerning steady $\mathfrak{m}$-pole solutions of the MS equation.

and flow field are written as

$$
\left\{u_{a}, p_{a}, F, U, V, P\right\}=\left\{u_{a}^{S}, 0, F^{S}, U^{S}, V^{S}, P^{S}\right\}+\left\{\tilde{u}_{a}, \tilde{p}_{a}, \tilde{F}, \tilde{U}, \tilde{V}, \tilde{P}\right\},
$$

where the 'steady velocity' $u_{a}^{S}=\frac{1}{2} q \overline{\left(\partial F^{S} / \partial \eta\right)^{2}}$ for $\xi>0$, and zero otherwise. As in Pelcé \& Rochwerger (1992), the perturbation consists also of an acoustic fluctuation, $\tilde{u}_{a}$ and $\tilde{p}_{a}$. The latter satisfies (2.29) and the linearised jump conditions

$$
\llbracket \tilde{p}_{a} \rrbracket_{-}^{+}=0, \quad \llbracket \tilde{u}_{a} \rrbracket_{-}^{+}=q \overline{\frac{\partial F^{S}}{\partial \eta} \frac{\partial \tilde{F}}{\partial \eta}} \equiv \tilde{\mathscr{J}}_{a}
$$

as well as the boundary conditions

$$
\tilde{u}_{a}(-\sigma L, \tau)=0, \quad \tilde{p}_{a}((1-\sigma) L, \tau)=0 .
$$

Following our previous notation, in what follows we shall write

$$
\left\{\mathscr{J}_{a}, \mathscr{B}_{a}\right\}=\left\{\mathscr{J}_{a}^{S}, 0\right\}+\left\{\tilde{\mathscr{J}}_{a}, \tilde{\mathscr{B}}_{a}\right\}
$$

where $\mathscr{J}_{a}^{S}=\llbracket u_{a}^{S} \rrbracket_{-}^{+}, \quad \tilde{\mathscr{J}}_{a}=\llbracket \tilde{u}_{a} \rrbracket_{-}^{+}$and $\tilde{\mathscr{B}}_{a}(\tau)=\llbracket \partial \tilde{p}_{a} / \partial \tilde{\xi} \rrbracket_{-}^{+}$. Inserting (3.5) into (2.31) and (2.32), we obtain the system governing the hydrodynamic field of the perturbations:

$$
\frac{\partial \tilde{U}}{\partial \xi}+\frac{\partial \tilde{V}}{\partial \eta}=0, \quad R \frac{\partial \tilde{U}}{\partial \tau}+\frac{\partial \tilde{U}}{\partial \xi}=-\frac{\partial \tilde{P}}{\partial \xi}, \quad R \frac{\partial \tilde{V}}{\partial \tau}+\frac{\partial \tilde{V}}{\partial \xi}=-\frac{\partial \tilde{P}}{\partial \eta},
$$

and the jump conditions

$$
[\tilde{U}]_{-}^{+}=0, \quad[\tilde{V}]_{-}^{+}=-q \frac{\partial \tilde{F}}{\partial \eta}, \quad[\tilde{P}]_{-}^{+}=-\left(F^{S} \tilde{\mathscr{B}}_{a}+\frac{q G}{1+q} \tilde{F}\right)
$$

Finally, from (2.33) follows the linearised flame-front equation:

$$
\frac{\partial \tilde{F}}{\partial \tau}=\tilde{U}\left(0^{-}, \eta, \tau\right)-\frac{\partial F^{S}}{\partial \eta} \frac{\partial \tilde{F}}{\partial \eta}+\delta M_{a} \frac{\partial^{2} \tilde{F}}{\partial \eta^{2}} .
$$


Since the coefficients of the system (3.9)-(3.11) are independent of the time $\tau$ and $\eta$, we may follow the standard method of stability analysis and seek normal modes,

$$
\left\{\tilde{U}, \tilde{V}, \tilde{P}, \tilde{F}, \tilde{u}_{a}, \tilde{p}_{a}\right\}=\left\{\mathfrak{U}(\xi, \eta), \mathfrak{V}(\xi, \eta), \mathfrak{P}(\xi, \eta), \mathfrak{F}(\eta), \mathfrak{u}_{\mathfrak{a}}(\tilde{\xi}), \mathfrak{p}_{\mathfrak{a}}(\tilde{\xi})\right\} \mathrm{e}^{\mathrm{i} \omega \tau}+\text { c.c. }
$$

where $\omega$ is allowed to be a complex number, with $-\operatorname{Im}(\omega)$ representing the growth rate, and c.c. stands for complex conjugate. If $\operatorname{Im}(\omega) \geqslant 0$, the system is linearly stable, while if $\operatorname{Im}(\omega)<0$, the system is linearly unstable. Inserting (3.12) into the system (3.6)-(3.11), one obtains the acoustic system in the frequency space,

$$
\left.\begin{array}{l}
\frac{\partial \mathfrak{u}_{\mathfrak{a}}}{\partial \tilde{\xi}}=-\mathrm{i} \omega \mathfrak{p}_{\mathfrak{a}}, \quad \frac{\partial \mathfrak{p}_{\mathfrak{a}}}{\partial \tilde{\xi}}=-R \mathbf{i} \omega \mathfrak{u}_{\mathfrak{a}}, \\
\llbracket \mathfrak{u}_{\mathfrak{a}} \rrbracket_{-}^{+}=q \frac{\overline{\partial F^{S}} \frac{\partial \mathfrak{F}}{\partial \eta}}{\partial \eta}, \quad \llbracket \mathfrak{p}_{\mathfrak{a}} \rrbracket_{-}^{+}=0, \quad \mathfrak{u}_{\mathfrak{a}}(-\sigma L)=0, \quad \mathfrak{p}_{\mathfrak{a}}((1-\sigma) L)=0,
\end{array}\right\}
$$

the corresponding hydrodynamic system (where the hat denotes the Fourier transform in $\eta$ ),

$$
\frac{\partial \widehat{\mathfrak{U}}}{\partial \xi}+\mathrm{i} k \widehat{\mathfrak{V}}=0, \quad R \mathrm{i} \omega \widehat{\mathfrak{U}}+\frac{\partial \widehat{\mathfrak{U}}}{\partial \xi}=-\frac{\partial \widehat{\mathfrak{P}}}{\partial \xi}, \quad R \mathrm{i} \omega \widehat{\mathfrak{V}}+\frac{\partial \widehat{\mathfrak{V}}}{\partial \xi}=-\mathrm{i} k \widehat{\mathfrak{P}},
$$

with the jump conditions

$$
[\widehat{\mathfrak{U}}]_{-}^{+}=0, \quad[\widehat{\mathfrak{V}}]_{-}^{+}=-q \mathrm{i} k \widehat{\mathfrak{F}}, \quad[\widehat{\mathfrak{P}}]_{-}^{+}=-\left(\widehat{F^{S}} \mathfrak{B}_{\mathfrak{a}}+\frac{q G}{1+q} \widehat{\mathfrak{F}}\right),
$$

and the flame-front equation,

$$
\mathrm{i} \omega \widehat{\mathfrak{F}}=\widehat{\mathfrak{U}}\left(0^{-}, k\right)-\left(\mathrm{i} k^{\prime} \widehat{F^{S}}\left(k^{\prime}\right)\right) \star\left(\mathrm{i} k^{\prime} \widehat{\mathfrak{F}}\left(k^{\prime}\right)\right)(k)-\delta M_{a} k^{2} \widehat{\mathfrak{F}}
$$

The perturbed acoustic system (3.13) can be solved analytically by inverting a $4 \times 4$ matrix depending on $\sigma$ and $\omega$. When $\Delta_{s}(\omega, \sigma) \neq 0$, where

$$
\Delta_{s}(\omega, \sigma) \equiv\left(\frac{R_{+}}{R_{-}}\right)^{1 / 2} \tan \left(R_{-}^{1 / 2} \omega \sigma L\right) \tan \left(R_{+}^{1 / 2} \omega(1-\sigma) L\right)-1,
$$

the matrix can be inverted to find $\mathfrak{u}_{\mathfrak{a}}$ and $\mathfrak{p}_{\mathfrak{a}}$ as well as the relation between $\mathfrak{B}_{\mathfrak{a}}=$ $\llbracket \partial \mathfrak{p}_{\mathfrak{a}} / \partial \tilde{\xi} \rrbracket_{-}^{+}$and $\mathfrak{J}_{\mathfrak{a}}=\llbracket \mathfrak{u}_{\mathfrak{a}} \rrbracket_{-}^{+}$:

$$
\mathfrak{B}_{\mathfrak{a}}=-\mathrm{i} \omega R_{+}\left\{1+q\left(1+\frac{1}{\Delta_{s}(\omega, \sigma)}\right)\right\} \mathfrak{J}_{\mathfrak{a}} .
$$

The acoustic dispersion relation corresponds to $\Delta_{s}(\omega, \sigma)=0$. The roots of this equation can be found numerically and represent the characteristic frequencies of the acoustic modes of the duct. It can be shown that there is an infinite (but discrete) set of characteristic frequencies $\omega_{j}$, and that they are real. The first six are given in table 1 . The coupling with the flame and hydrodynamics would render $\omega$ complex, but its real part remains close to one of the acoustic characteristic frequencies. 


$\begin{array}{lcccccr}\text { Acoustic modes } & \omega_{1} & \omega_{2} & \omega_{3} & \omega_{4} & \omega_{5} & \omega_{6} \\ \text { Non-dimensional } & 50.7 & 133.6 & 200.6 & 297.6 & 394.6 & 461.6 \\ \text { Frequency (Hz) } & 121.7 & 320.7 & 481.4 & 714.3 & 947.1 & 1107.9 \\ & & & & & & \\ \text { Parameters } & \sigma & \ell^{*}(\mathrm{~m}) & h^{*}(\mathrm{~m}) & M & q & \\ \text { Values } & 0.5 & 1.2 & 0.1 & 0.0007 & 5.25 & \end{array}$

TABLE 1. First six acoustic modes for a given set of parameters.

Solving (3.14) subject to (3.15), we may express the solution for the hydrodynamic field in terms of $\widehat{\mathfrak{F}}$, the details of which are relegated to appendix B. Substitution of the solution into (3.16) leads to a single equation for $\widehat{\mathfrak{F}}$,

$$
h_{1}(\omega, k)\left(\mathrm{i} k^{\prime} \widehat{F^{S}}\left(k^{\prime}\right)\right) \star\left(\mathrm{i} k^{\prime} \widehat{\mathfrak{F}}\left(k^{\prime}\right)\right)(k)+h_{2}(\omega, k) \widehat{\mathfrak{F}}+h_{3}(\omega, k ; \sigma) \mathfrak{J}_{\mathfrak{a}}=0
$$

where

$$
\begin{aligned}
h_{1}(\omega, k) & =\left(\mathrm{i} \omega R_{+}+|k|\right)+\left(|k|+\mathrm{i} \omega R_{-}\right), \\
h_{2}(\omega, k) & =h_{1}(\omega, k)\left(\mathrm{i} \omega+\delta M_{a} k^{2}\right)+|k| q\left(\frac{G}{1+q}-|k|\right), \\
h_{3}(\omega, k ; \sigma) & =-\mathrm{i} \omega|k| R_{+}\left\{1+q\left(1+\frac{1}{\Delta_{s}(\omega, \sigma)}\right)\right\} \widehat{F^{S}} .
\end{aligned}
$$

Equation (3.19) forms the eigenvalue problem that will allow us to determine $\omega$.

As in appendix A, for a flame in a duct, the Fourier transforms are interpreted as truncated Fourier series. Note, however, that $\mathfrak{F}$ does not have to be real, but it still needs to be even with respect to $\eta$, which implies that $\widehat{\mathfrak{F}}_{n}=\widehat{\mathfrak{F}}_{-n}$. Using the definition of space average and convolution, one can show that

$$
\begin{gathered}
\mathfrak{J}_{\mathfrak{a}}=q \frac{\overline{\partial F^{S}} \frac{\partial \mathfrak{F}}{\partial \eta}}{\partial \eta}=2 q \sum_{m=1}^{N} m^{2} \widehat{F}_{m}^{S} \widehat{\mathfrak{F}}_{m}, \\
\left(\mathrm{i} k \widehat{F^{S}}\right) \star(\mathrm{i} k \widehat{\mathfrak{F}})(n)=-\sum_{m=1}^{N}\left(m(n-m) \widehat{F}_{n-m}^{S}-m(n+m) \widehat{F}_{n+m}^{S}\right) \widehat{\mathfrak{F}}_{m},
\end{gathered}
$$

and so that the discrete version of (3.19) can be written as a system of $N$ equations,

$$
\begin{aligned}
0= & -h_{1}(\omega, n) \sum_{m=1}^{N}\left(m(n-m) \widehat{F}_{n-m}^{S}-m(n+m) \widehat{F}_{n+m}^{S}\right) \widehat{\mathfrak{F}}_{m} \\
& +h_{2}(\omega, n) \widehat{\mathfrak{F}}_{n}+2 q h_{3}(\omega, n ; \sigma) \sum_{m=1}^{N} m^{2} \widehat{F}_{m}^{S} \widehat{\mathfrak{F}}_{m} \quad(1 \leqslant n \leqslant N) .
\end{aligned}
$$

By introducing a vectorial representation $f$ of the Fourier coefficients $\widehat{\mathfrak{F}}_{m}$ of $\mathfrak{F}$, such that $\boldsymbol{f}=\left(\widehat{\mathfrak{F}}_{1}, \ldots, \widehat{\mathfrak{F}}_{N}\right)^{\mathrm{T}}$, the equation $(3.25)$ can be recast into the matrix form,

$$
\boldsymbol{A}(\omega, \sigma) \boldsymbol{f}=0,
$$

where $\boldsymbol{A}$ is an $N \times N$ matrix whose entries are nonlinear functions of $\omega$. Hence (3.26) is a nonlinear non-polynomial (because of $\Delta_{s}(\omega ; \sigma)$ ) eigenvalue problem, which is 
difficult to solve. In order to ease the task, we assume that $\omega$ remains close to the first acoustic mode $\omega_{1}$ defined by $\Delta_{s}\left(\omega_{1} ; \sigma\right)=0$, and so write

$$
\omega=\omega_{1}(\sigma)+\tilde{\omega}, \quad \text { where } \tilde{\omega} \ll \omega_{1} .
$$

The function $\Delta_{s}(\omega, \sigma)$ is then approximated by its Taylor expansion, $\Delta_{s}(\omega, \sigma)=$ $\Delta_{s}^{\prime}\left(\omega_{1}, \sigma\right) \tilde{\omega}$, and hence the problematic term $2 q h_{3}(\omega, n ; \sigma)$ simplifies to

$$
2 q h_{3}(\omega, n ; \sigma) \approx h_{4}(n)-h_{5}(\sigma, n) / \tilde{\omega},
$$

where

$$
h_{4}(n)=-2 \mathrm{i} q \omega_{1}|n| \widehat{F}_{n}^{S} \quad \text { and } \quad h_{5}(\sigma, n)=2 \mathrm{i} q^{2} \omega_{1}|n| R_{+} \widehat{F}_{n}^{S} / \Delta_{s}^{\prime}\left(\omega_{1}, \sigma\right) .
$$

As a result, the system of $N$ equations (3.25) can be simplified to

$$
\begin{aligned}
h_{5}(\sigma, n) \sum_{m=1}^{N} m^{2} \widehat{F}_{m}^{S} \widehat{\mathfrak{F}}_{m}= & \tilde{\omega}\left\{-h_{1}\left(\omega_{1}, n\right) \sum_{m=1}^{N}\left(m(n-m) \widehat{F}_{n-m}^{S}-m(n+m) \widehat{F}_{n+m}^{S}\right) \widehat{\mathfrak{F}}_{m}\right. \\
& \left.+h_{2}\left(\omega_{1}, n\right) \widehat{\mathfrak{F}}_{n}+h_{4}(n) \sum_{m=1}^{N} m^{2} \widehat{F}_{m}^{S} \widehat{\mathfrak{F}}_{m}\right\}
\end{aligned}
$$

which can be written as a generalised linear eigenvalue problem of the standard form,

$$
\boldsymbol{B}(\sigma) \boldsymbol{f}=\tilde{\omega} \boldsymbol{C} \boldsymbol{f},
$$

where $\boldsymbol{B}(\sigma)$ and $\boldsymbol{C}$ are $N \times N$ matrices. For each value of $\sigma$, the eigenvalue problem (3.31) can be solved numerically and the results are presented in the next subsection.

\subsection{Results}

It is worth noting first that, if the hydrodynamic perturbation is treated as being quasisteady and acoustic fluctuations are ignored, all the troublesome nonlinear terms in $\boldsymbol{A}$ disappear and the problem (3.26) reduces to a linear eigenvalue problem, which can easily be solved numerically. As part of the validation of our code, we solved this reduced linear eigenvalue problem for one- and two-pole solutions at two different values of the parameter $\gamma$. In these cases, all the eigenvalues have a positive imaginary part $\omega_{i}>0$, which means that these steady solutions are both stable as predicted by the theory of Vaynblat \& Matalon (2000a).

The eigenvalue problem (3.31) is solved for $\gamma=2.1$ and $\gamma=6.2$. In each case, the eigenvalues are calculated for $\sigma \in[0,1]$. The largest growth rate is plotted in figure 5. As is illustrated, for whatever value of $\sigma \in[0,1]$, there is always at least one eigenvalue with $\omega_{i}<0$; the growth rate reaches a maximum around $\sigma \approx 0.3$. Overall, the growth rates for $\gamma=6.2$ are one order of magnitude bigger than those for $\gamma=2.1$. In figure 6 , the eigenfunction $F_{\text {eig }}$ of the most unstable mode for $\sigma=0.5$ is plotted. For $\gamma=2.1$, the eigenfunction is relatively simple, exhibiting two peaks, whereas for $\gamma=6.2, F_{\text {eig }}$ is highly oscillatory.

Clearly, when the acoustic and hydrodynamic variations are considered, the one-pole and two-pole solutions are actually linearly unstable. This is an important result since it implies that the spontaneous acoustic field cannot be ignored when considering combustion problems. Interestingly enough, the growth rates have a similar profile to that found in Pelcé \& Rochwerger (1992). In particular, the position of the peak growth around $\sigma \approx 0.3$ is the same as in figure 6 of Pelcé \& Rochwerger (1992). 

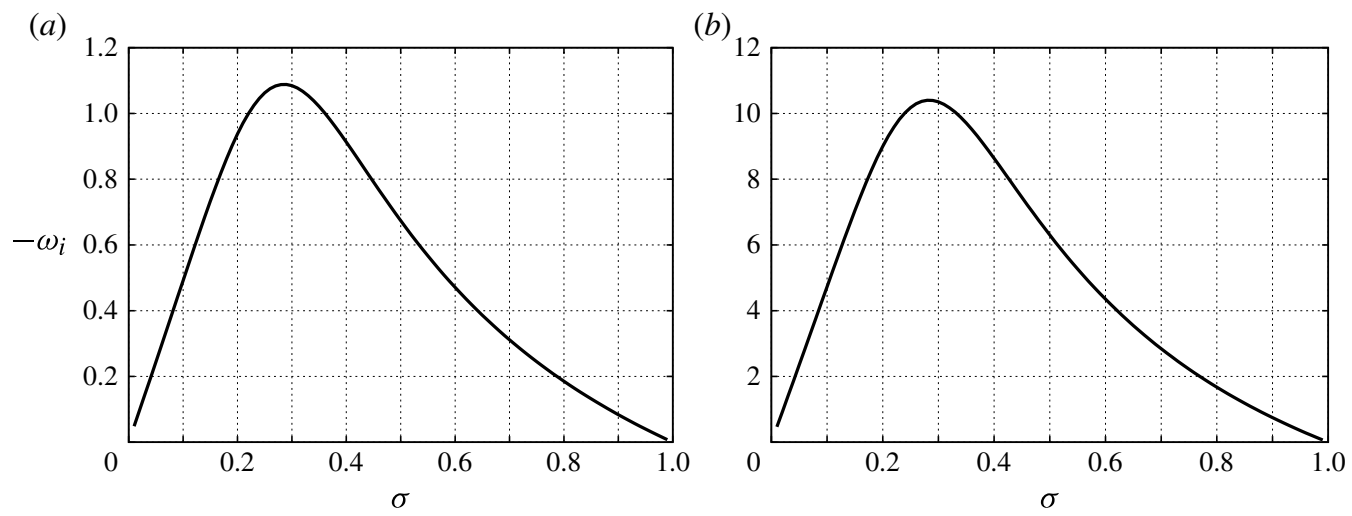

FIGURE 5. Stability results for the full model for $(a) \gamma=2.1$ and $(b) \gamma=6.2$.
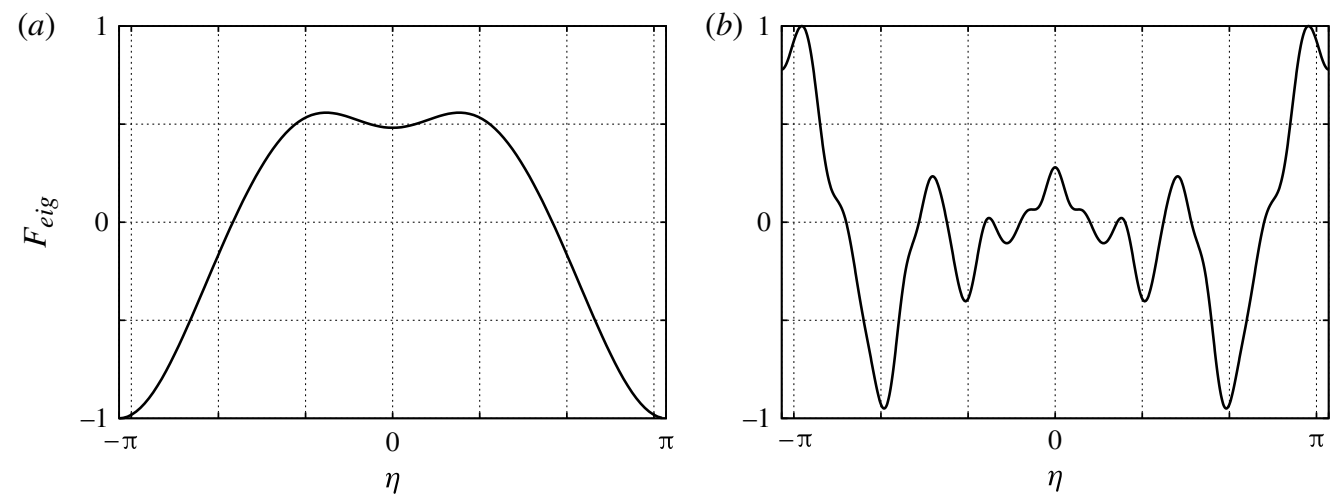

FIGURE 6. Normalised eigenfunction $F_{\text {eig }}$ corresponding to the most unstable eigenvalue for $\sigma=0.5$ for $(a) \gamma=2.1$ and $(b) \gamma=6.2$.

There is therefore a good qualitative agreement, but we do not expect a quantitative agreement for two main reasons. Firstly, in this work the steady state used for stability analysis is an exact solution of the flame equation, while in Pelcé \& Rochwerger (1992) it is artificially chosen as a cosine function. Secondly, gravity and some $O(\delta)$ terms have so far been neglected in our calculations, while they were retained in Pelcé \& Rochwerger (1992).

The results obtained thus far are for $M=0.0007$ and $q=5.25$. It is of interest to study how the value and the location of the maximum growth rate vary with $M$ and $q$. The results are presented in figure 7 . For any $q>0$, the curved flame is unstable. The growth rate increases with the heat release $q$, whilst the location of the peak growth seems to move further down the duct. The Mach number $M$ does not appear to have such an important effect: the growth rate decreases only slightly as $M$ increases, while the location of the maximum remains approximately the same.

\section{Weakly nonlinear evolution of the flame and its spontaneous sound}

In order to validate the linear instability results of the previous section and to understand more about the weakly nonlinear effects, we will formulate an initial-value problem governing the nonlinear interaction and evolution of the flame and the 
(a)

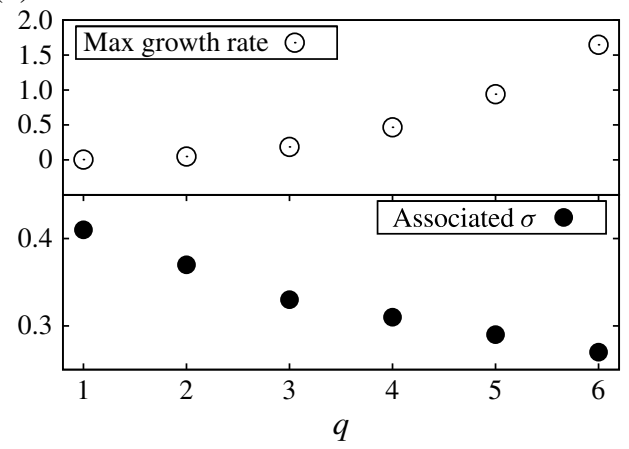

(b)

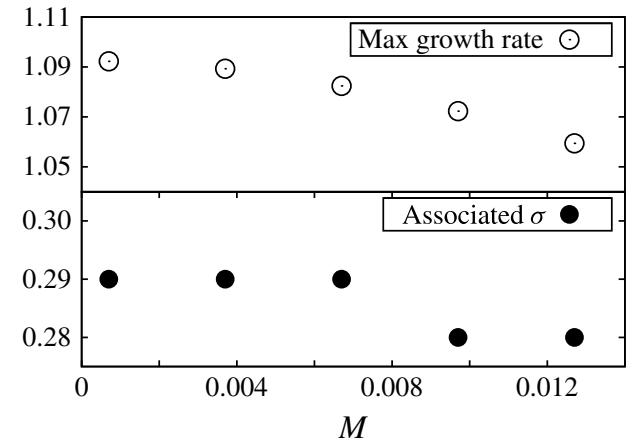

FIGURE 7. Variation of the maximum growth rate $(a)$ with $q$ at $M=0.0007$ and $(b)$ with $M$ for $q=5.25$. Both graphs are obtained for $\gamma=2.1$.

spontaneous acoustic field. The present theoretical development is motivated in particular by the need to provide a better description of the phenomena observed in the experiments of Markstein (1953) and Searby (1992). They observed that a flame propagating in a tube may wrinkle whilst sound waves are generated and amplified. After attaining a certain magnitude, the sound wave may inhibit wrinkling and then saturate in the case of relatively slow (weak) flames. For fast (strong) flames, the sound wave induces more rapid wrinkling, which in turn leads to an almost explosive amplification of sound. These two regimes have usually been referred to as primary and secondary (subharmonic) parametric instabilities. Neither of them can be predicted by the usual $G$ equation approach because it only accounts for the kinematic advection of the flame, but that effect merely makes a freely propagating flame vibrate rigidly. The parametric instability theory for an externally imposed sound wave (Markstein \& Squire 1955; Searby \& Rochwerger 1991, and others), and the stability analysis of a specified steady curved flame (as was done in Pelcé \& Rochwerger (1992) and the previous section of the present paper) have revealed two crucial mechanisms and thus explain some key aspects of the phenomena. They do not, however, provide a complete description because in experiments the flame and sound are both evolving and in mutual interaction. The two mechanisms operate simultaneously and dynamically rather than being in isolation and 'static' as was treated in the analyses. It is clear that a better model must be able to describe the generation of the spontaneous sound and its two-way dynamic coupling with the flame. From the simplified system in $\$ 2.3$, we will derive such a model.

\subsection{The evolution system}

With the hydrodynamic field being linearised, its solution can be expressed in terms of the flame-front function by using Fourier analysis as in appendices A and B. The system (2.31)-(2.33) can then be reduced to a single equation in spectral space. The derivation is rather tedious, and is relegated to appendix C. Here we only present the equation, which for a two-dimensional flame reads

$$
\begin{aligned}
A \frac{\partial^{2} \widehat{F}}{\partial \tau^{2}}+B(k) \frac{\partial \widehat{F}}{\partial \tau}+C(k, \tau) \widehat{F}= & -|k|\left(\mathrm{i} k^{\prime} \widehat{F}\left(k^{\prime}\right)\right) \star\left(\mathrm{i} k^{\prime} \widehat{F}\left(k^{\prime}\right)\right)(k) \\
& -A\left(\mathrm{i} k^{\prime} \widehat{F}\left(k^{\prime}\right)\right) \star\left(\mathrm{i} k^{\prime} \frac{\partial \widehat{F}}{\partial \tau}\left(k^{\prime}\right)\right)(k),
\end{aligned}
$$


where

$$
\begin{aligned}
& \left.\begin{array}{l}
A=R_{+}+R_{-}, \quad B(k)=A \delta M_{a} k^{2}+2|k|, \\
C(k, \tau)=-q k^{2}+|k|\left[\mathscr{B}_{a}(\tau)+q G /(1+q)\right]+2 \delta M_{a}|k|^{3} .
\end{array}\right\}
\end{aligned}
$$

The left-hand side of (4.1) has the same structure as the equations in Markstein \& Squire (1955), Searby \& Rochwerger (1991) and Wu \& Law (2009). The only difference from the latter two is that we have omitted some $O(\delta)$ terms in the coefficients $A, B, C$. This level of approximation is appropriate since we are interested in the qualitative behaviour of the dynamically coupled flame and spontaneous sound, rather than in the quantitative prediction of the well-understood parametric instability.

On (4.1), we impose the initial condition

$$
\widehat{F}(k, 0)=\widehat{F}_{0}(k), \quad \frac{\partial \widehat{F}}{\partial \tau}(k, 0)=0,
$$

where $\widehat{F}_{0}$ is the Fourier transform of $F_{0}$, the initial flame profile. In our computation, $F_{0}$ is chosen to be close to one of the steady solutions, namely,

$$
F_{0}=F^{S}+\epsilon \cos \left(N_{\text {pert }} \eta\right) \quad \text { or } \quad F_{0}=F^{S}+\epsilon F_{\text {eig }},
$$

where $\epsilon$ is a small parameter, $N_{\text {pert }}$ is an integer and $F_{\text {eig }}$ represents the eigenfunction associated with the most unstable eigenvalue obtained from the linear stability analysis of $\S 3$. The flame-front equation is coupled to the acoustic equations (2.34) through (2.35) and $\mathscr{B}_{a}$ in (4.2). The present interactive system, which will be referred to as the 'coupled flame-acoustic model', may be viewed as an extension of the MS equation by accounting for the spontaneous acoustic field, its back-effect, as well as the unsteadiness of the hydrodynamic field. The algorithm for solving the interactive evolution system consists of two modules, an acoustic solver and a flame-front solver, which are described below.

\subsection{Numerical resolution of the acoustic system}

\subsubsection{Semi-analytical method of characteristics}

The system (2.34) governing the acoustic fluctuations can be rewritten as

$$
\left(p_{a}\right)_{\tau}+\left(u_{a}\right)_{\tilde{\xi}}=0, \quad\left(u_{a}\right)_{\tau}+c^{2}\left(p_{a}\right)_{\xi}=0,
$$

for $\tilde{\xi} \in\left[L^{-}, L^{+}\right]$, with $L^{-}=-\sigma L<0$ and $L^{+}=(1-\sigma) L>0$, where

$$
c= \begin{cases}c^{-}=\sqrt{1 / R_{-}} & \text {if } \tilde{\xi}<0, \\ c^{+}=\sqrt{1 / R_{+}} & \text {if } \tilde{\xi}>0 .\end{cases}
$$

The solution is subject to the boundary and jump conditions

$$
u_{a}\left(L^{-}, \tau\right)=0, \quad p_{a}\left(L^{+}, \tau\right)=0 ; \quad \llbracket u_{a} \rrbracket_{-}^{+}=\mathscr{J}_{a}(\tau), \quad \llbracket p_{a} \rrbracket_{-}^{+}=0 .
$$

Throughout $\S 4.2$, the jump $\mathscr{J}_{a}(\tau)$ is considered known for all $\tau$. Eliminating $u_{a}$ between the two equations in (4.5), we obtain the wave equation

$$
\left(p_{a}\right)_{\tau \tau}-c^{2}\left(p_{a}\right)_{\tilde{\xi} \tilde{\xi}}=0,
$$


which has a discontinuous coefficient. The equation can be solved by the method of characteristics to obtain the general solution,

$$
p_{a}(\tilde{\xi}, \tau)= \begin{cases}A_{c h}\left(\tau+\tilde{\xi} / c^{-}\right)+C_{c h}\left(\tau-\tilde{\xi} / c^{-}\right) & \text {if } \tilde{\xi}<0 \\ B_{c h}\left(\tau-\tilde{\xi} / c^{+}\right)+D_{c h}\left(\tau+\tilde{\xi} / c^{+}\right) & \text {if } \tilde{\xi}>0\end{cases}
$$

where $A_{c h}, B_{c h}, C_{c h}$ and $D_{c h}$ are unknown functions of one variable. The solution can be interpreted as the superposition of two waves travelling in opposite directions. The conditions (4.7), when combined with the representation (4.9) and the equations (4.5), allow one to express $C_{c h}$ and $D_{c h}$ in terms of $A_{c h}$ and $B_{c h}$. Moreover, one obtains the following matrix representation for $A_{c h}$ and $B_{c h}$ :

$$
\left(\begin{array}{cc}
1 & -1 \\
c^{-} & c^{+}
\end{array}\right)\left(\begin{array}{l}
A_{c h}(\tau) \\
B_{c h}(\tau)
\end{array}\right)=\left(\begin{array}{cc}
-1 & -1 \\
c^{-} & -c^{+}
\end{array}\right)\left(\begin{array}{l}
A_{c h}\left(\tau-\tau^{-}\right) \\
B_{c h}\left(\tau-\tau^{+}\right)
\end{array}\right)+\left(\begin{array}{c}
0 \\
\mathscr{J}_{a}(\tau)
\end{array}\right)
$$

where $\tau^{ \pm}= \pm 2 L^{ \pm} / c^{ \pm}$is the time taken by a wave to propagate twice along the right(left)-hand side of the acoustic domain, respectively. Hence, as long as $\mathscr{J}_{a}$ is given, if one knows the values of $A_{c h}$ and $B_{c h}$ at previous times, it is possible to recover their values at the current time. As an alternative to the method of characteristics, a full numerical approach, the modified immersed interface method (MIIM), is presented in appendix D.

\subsubsection{Initial acoustic conditions and validation}

For both methods described in $\S 4.2 .1$ and appendix D, the initial conditions must be chosen carefully. Indeed, the equations do not allow for initial conditions with both pressure and velocity being zero. Hence, an appropriate initial profile had to be specified to ensure a smooth solution. We chose a simple initial profile close to the steady behaviour of the acoustic pressure and velocity described in $\S \S 2.2$ and 3.2, given by

$$
u_{a}(\tilde{\xi}, 0)=\left\{\begin{array}{ll}
0 & \text { if } \tilde{\xi}<0, \\
\mathscr{J}_{a}(0) & \text { if } \tilde{\xi}>0,
\end{array} \quad \text { and } \quad p_{a}(\tilde{\xi}, 0)= \begin{cases}0 & \text { if } \tilde{\xi}<0 \\
0 & \text { if } \tilde{\xi}>0\end{cases}\right.
$$

For validation, both methods (characteristics and MIIM) have been implemented for different given functions $\mathscr{J}_{a}(\tau)$ and the results are found to agree. Of course, being semi-analytical, the characteristics method is much faster than the MIIM and does not have a Courant-Friedrichs-Lewy (CFL) restriction. However, the MIIM has the possibility to be extended to higher dimensions if necessary. In summary, if the function $\mathscr{J}_{a}(\tau)$ is known for all time, it is possible to solve the acoustic problem using either method.

\subsection{Numerical resolution of the spectral flame equation}

We now consider the numerical resolution of (4.1) and (4.2). To adopt standard notation in numerical analysis, we introduce

$$
\boldsymbol{y}(k, \tau)=\left(\widehat{F}(k, \tau), \frac{\partial \widehat{F}}{\partial \tau}(k, \tau)\right)^{\mathrm{T}} \equiv\left(y_{1}(k, \tau), y_{2}(k, \tau)\right)^{\mathrm{T}} .
$$


The initial-value problem (4.1) and (4.2) can then be recast to a first-order system,

$$
\boldsymbol{y}^{\prime}=\boldsymbol{M}(k, \tau, \boldsymbol{y}), \quad \boldsymbol{y}(k, 0)=\left(\widehat{F}_{0}(k), 0\right)^{\mathrm{T}},
$$

where $\boldsymbol{M}(k, \tau, \boldsymbol{y})=\left[\boldsymbol{M}_{1}(k, \tau) \boldsymbol{y}+\boldsymbol{M}_{2}(k, \boldsymbol{y})\right] / A$ with

$$
\begin{gathered}
\boldsymbol{M}_{1}=\left(\begin{array}{cc}
0 & A \\
-C(k, \tau) & -B(k)
\end{array}\right), \\
\boldsymbol{M}_{2}=\left(\begin{array}{c}
0 \\
-|k|\left\{\mathrm{i} k^{\prime} y_{1}\left(k^{\prime}, \tau\right)\right\} \star\left\{\mathrm{i} k^{\prime} y_{1}\left(k^{\prime}, \tau\right)\right\}-A\left\{\mathrm{i} k^{\prime} y_{1}\left(k^{\prime}, \tau\right)\right\} \star\left\{\mathrm{i} k^{\prime} y_{2}\left(k^{\prime}, \tau\right)\right\}
\end{array}\right) .
\end{gathered}
$$

Let $\boldsymbol{y}_{n}$ denote a numerical approximation of $\boldsymbol{y}\left(k, \tau_{n}\right)$. The system (4.13) is discretised by using an explicit fourth-order Adams-Bashforth (AB4) finite-difference scheme:

$$
\boldsymbol{y}_{n+1}=\boldsymbol{y}_{n}+\frac{\Delta \tau}{12}\left(23 \boldsymbol{M}\left(k, \tau_{n}, \boldsymbol{y}_{n}\right)-16 \boldsymbol{M}\left(k, \tau_{n-1}, \boldsymbol{y}_{n-1}\right)+5 \boldsymbol{M}\left(k, \tau_{n-2}, \boldsymbol{y}_{n-2}\right)\right) \text {. }
$$

At each time step $\tau_{n}=n \Delta \tau$, it is necessary to evaluate $\boldsymbol{M}$, which requires the value of $\mathscr{B}_{a}$ and involves computing the convolutions appearing in $\boldsymbol{M}_{2}$. In this particular module of the algorithm, we assume that $\mathscr{B}_{a}(\tau)$ is known for all time. The convolutions are computed by using the fast Fourier transform (FFT) and the inverse fast Fourier transform (IFFT) algorithms. As is outlined in Trefethen (2000), numerical stability of such a spectral scheme is generally subject to a restriction of the type $\Delta \tau<\alpha / N^{2}$, where $N$ is the number of points used to discretise the flame in physical and spectral spaces. In our case, numerical experimentation suggests that the scheme is stable for $\alpha<1.49$, and in practice we used $\Delta \tau=1 / N^{2}$. For example, if the flame is described by 256 points, this leads to a time step $\Delta \tau \approx 1.5 \times 10^{-5}$. Throughout the scheme, we make use of the ' $2 / 3$ rule' (Orszag 1971) in order to avoid aliasing. In order to test the method, we set $\mathscr{B}_{a}(\tau) \equiv 0$, and recovered the expected steady states; the evolution towards the steady states is found to be independent of the choice of $\Delta \tau$. Hence, if $\mathscr{B}_{a}(\tau)$ is known for all time, it is possible to solve the spectral flame equation numerically.

\subsection{Numerical resolution of the coupled acoustic-flame model}

The two modules presented in $\S \S 4.2$ and 4.3 are now linked to obtain a coupled numerical scheme solving the overall problem. At each time step, this requires evaluating $\mathscr{J}_{a}$ using the solution for the flame front, and $\mathscr{B}_{a}$ using the solution for the acoustic field. For the first task, it follows from (2.35) and the definition of convolution that

$$
\mathscr{J}_{a}(\tau)=\frac{1}{2} q \overline{(\tilde{\nabla} F)^{2}}=\frac{1}{2} q\left(\mathrm{i} k^{\prime} \widehat{F}\left(k^{\prime}, \tau\right)\right) \star\left(\mathrm{i} k^{\prime} \widehat{F}\left(k^{\prime}, \tau\right)\right)(0, \tau),
$$

which means that, once the convolutions are computed for all values of $k$ using the method of FFT and IFFT as described in $\S 4.3$, we obtain $\mathscr{J}_{a}$ by simple evaluation at $k=0$. This noted, the coupled method should be implemented as illustrated in figure 8 . Let us assume that we know the solution $\boldsymbol{y}_{n}, \boldsymbol{M}\left(k, \tau_{n-1}, \boldsymbol{y}_{n-1}\right)$ and $\boldsymbol{M}\left(k, \tau_{n-2}, \boldsymbol{y}_{n-2}\right)$. The first step consists of computing the two convolutions involved in (4.15). These convolutions are used to obtain $\boldsymbol{M}_{2}$, and the evaluation of the first one also determines $\mathscr{J}_{a}\left(\tau_{n}\right)$ through (4.17). The acoustic solver is then used to obtain $\mathscr{B}_{a}\left(\tau_{n}\right)$. At this stage, we have enough information to obtain $\boldsymbol{M}\left(k, \tau_{n}, \boldsymbol{y}_{n}\right)$, which allows us to march in time using AB4 and obtain $\boldsymbol{y}_{n+1}$. This new solution is then fed again into the algorithm, and the process is repeated. Note that, for simplicity, we have presented an algorithm involving the method of characteristics. However, a similar but more complicated algorithm involving the MIIM has also been developed and implemented. 


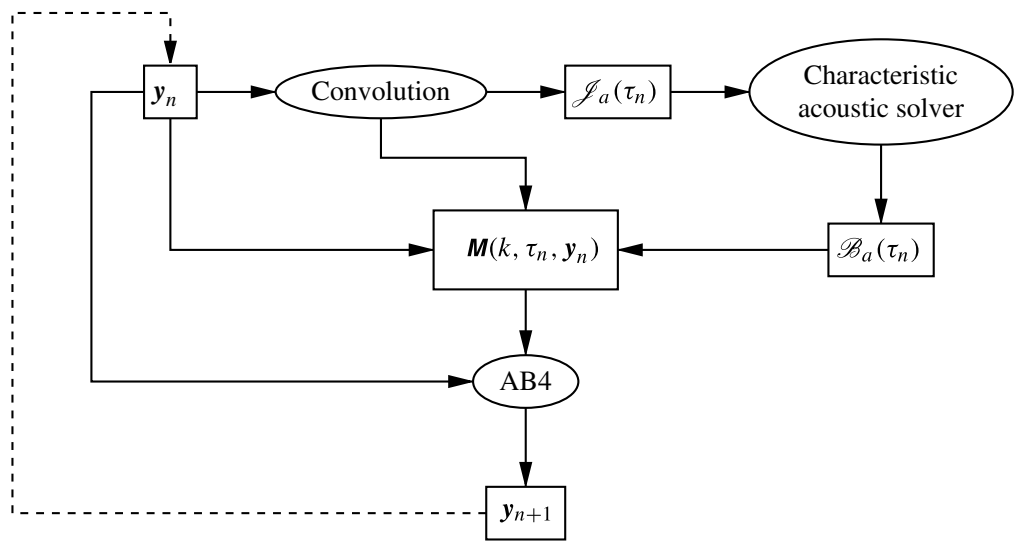

FIGURE 8. Flow diagram of the coupled algorithm.

$\begin{array}{lccccccc}\text { Parameters } & \sigma & M & U_{L}\left(\mathrm{~m} \mathrm{~s}^{-1}\right) & q & \ell^{*}(\mathrm{~m}) & h^{*}(\mathrm{~m}) & G \\ \text { Values } & 0.5 & 0.0007 & 0.24 & 5.25 & 1.2 & 0.1 & 0\end{array}$

TABLE 2. Parameter values used in $\S \S 5.1$ and 5.2.

\section{Numerical results}

\subsection{Two representative cases}

The numerical results in $\S \S 5.1$ and 5.2 are given for the parameter values listed in table 2, while two different values of $\gamma$ are chosen, $\gamma=2.1$ and $\gamma=6.2$, corresponding to cusped steady states $F^{S}$ being one-pole and two-pole solutions, respectively. For the case $\gamma=2.1$, the initial flame profile is chosen as $F_{0}=F^{S}+\epsilon \cos \left(N_{\text {pert }} \eta\right)$ with $\epsilon=0.05$ and $N_{\text {pert }}=10$, while for the case $\gamma=6.2, F_{\text {eig }}$ is used to perturb the flame with $\epsilon=0.05$; see (4.4). In most calculations, we use $N=64$ and $\Delta \tau=1 / N^{2}$. Refining the resolution to $N=128$ does not cause any appreciable difference to the results. Figure 9 displays the flame shapes at different times. For $\gamma=2.1$, the flame remains close to the steady state for a reasonably long time, and then tends to flatten, finally reaching an almost perfectly flat state. Here we terminated the computation at $\tau=16$, but further increase of $\tau$ does not change the flat state of the flame. If figure 9 was made into a movie, one could notice that, while becoming more flat overall, some small oscillations of the flame shape occur. For $\gamma=6.2$, we observe a similar tendency: the flame remains in the vicinity of the cusped steady state for a while before starting to flatten. However, when approaching the flat state, wrinkling appears, i.e. the flame becomes cellular. The amplitude of the wrinkling then grows exponentially with time. This behaviour is consistent with some of the fully numerical results presented in Gonzalez (1996).

In order to gain a better insight into the phenomena, we examine the evolution of the corresponding spontaneous acoustic field, represented by the pressure at the inlet of the duct. As is shown in figure 10, in both cases, we observe a first exponential growth of the pressure during the earlier phase when the flame is deviating from the steady state (cf. figure 9). As will be seen below, this corresponds precisely to the linear instability described in $\S 3.3$. Beyond this, in both cases, the pressure tends to saturate owing to nonlinear effects. In the case of $\gamma=2.1$, a regime corresponding 

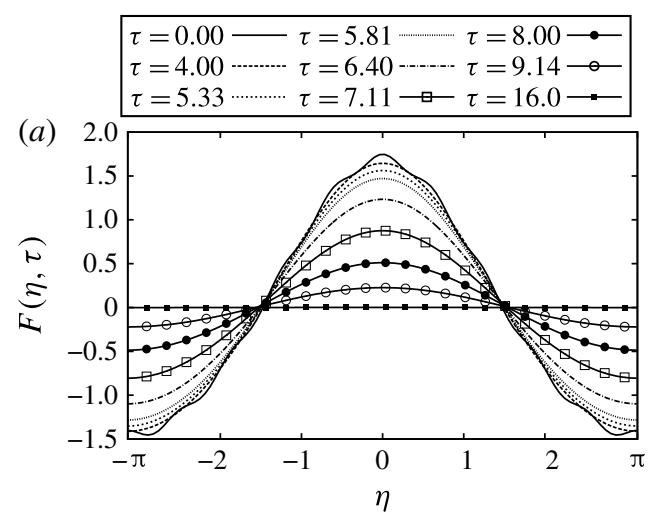

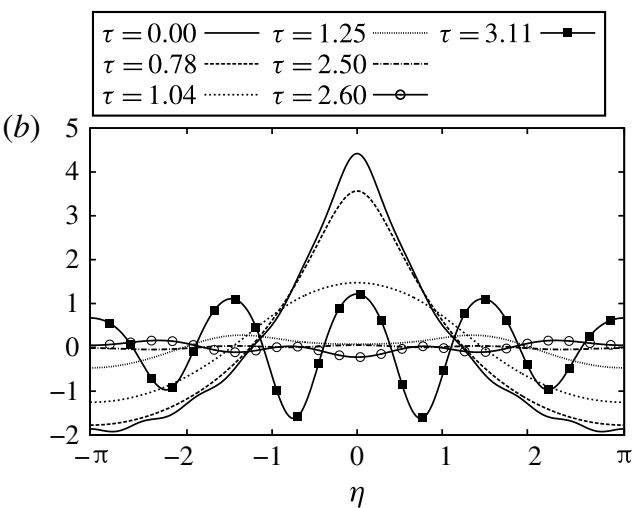

FIGURE 9. Evolution of the flame shape for $(a) \gamma=2.1$ and $(b) \gamma=6.2$.
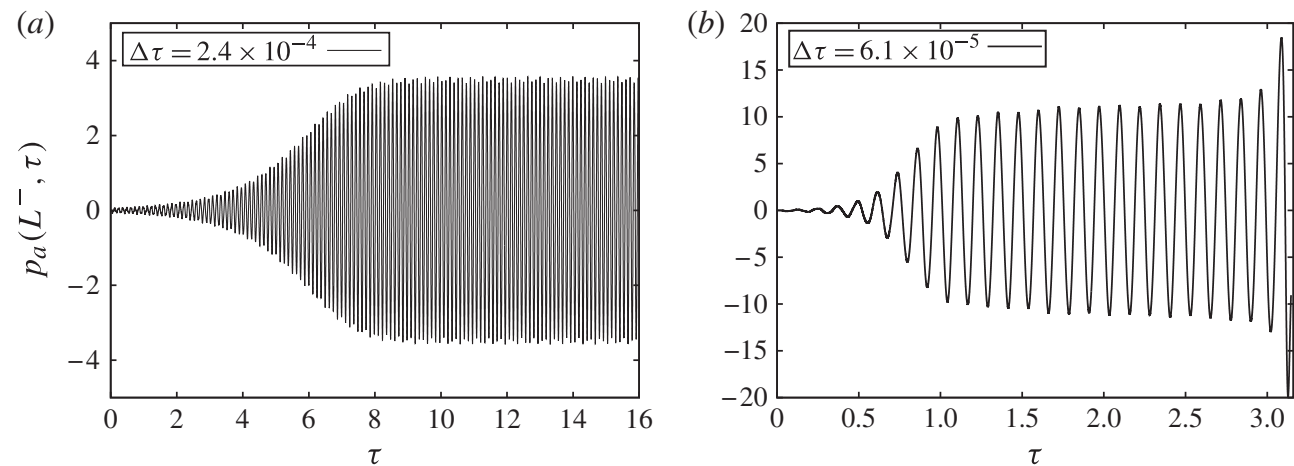

FIgURE 10. Evolution of the acoustic pressure at the inlet of the duct for $(a) \gamma=2.1$ and $(b) \gamma=6.2$.

to a limit cycle is reached. (Of course, if the calculation is run for a very long time, the amplitude of the limit cycle tends to decrease slightly owing to numerical dissipation.) In the case of $\gamma=6.2$, however, the saturated state does not persist, and instead a second exponential growth occurs, leading to a strongly nonlinear regime. We will see in $\S 5.2 .2$ that this corresponds to a subharmonic parametric instability. A time-spectral analysis of the pressure signal shows that, while recovering the theoretical acoustic frequencies of the duct, the signal is largely dominated by the first characteristic frequency of the duct, $\omega_{1}$, given in table 1 . This supports the approximation of linearising around this particular mode made in $\S 3.2$. In order to understand the behaviour of the flame, another important quantity to monitor is $\mathscr{B}_{a}(\tau)$, which represents the back-action due to acoustic acceleration. As is illustrated by figure 11, it has a very similar behaviour to the acoustic pressure, but with a much larger amplitude, estimated to be between 130 and 150 for the case $\gamma=2.1$ and between 430 and 450 for the case $\gamma=6.2$. It is also of interest to observe (see figure 12) the evolution of the acoustic velocity jump $\mathscr{J}_{a}(\tau)$. In the case of $\gamma=2.1$, $\mathscr{J}_{a}$ diminishes in an oscillatory manner. At large times, $\mathscr{J}_{a} \approx 0$, even though a closer examination (i.e. the zoomed view around $\tau \approx 11$ ) indicates that small oscillations are present. A rather interesting interpretation of these numerical results is that a flat flame, which is intrinsically unstable in a silent environment due to DL instability, 
(a)

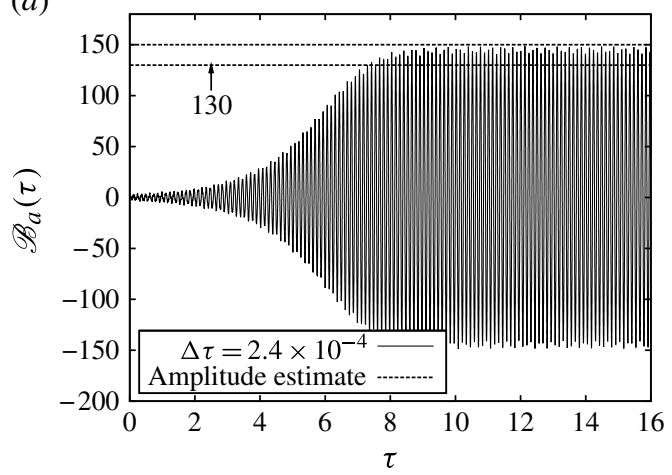

(b)

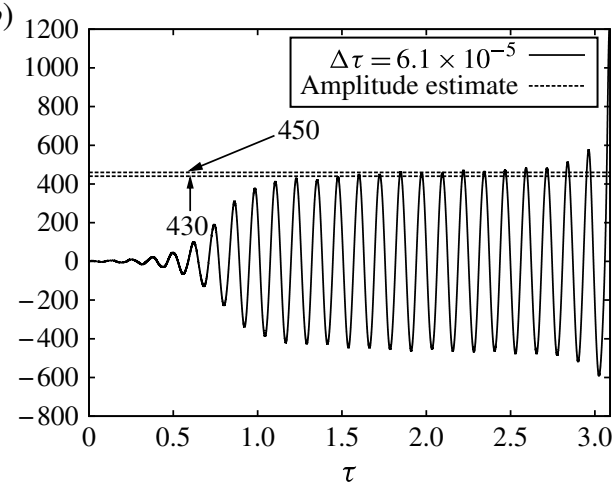

FIGURE 11. Evolution of $\mathscr{B}_{a}(\tau)$ for $(a) \gamma=2.1$ and $(b) \gamma=6.2$.

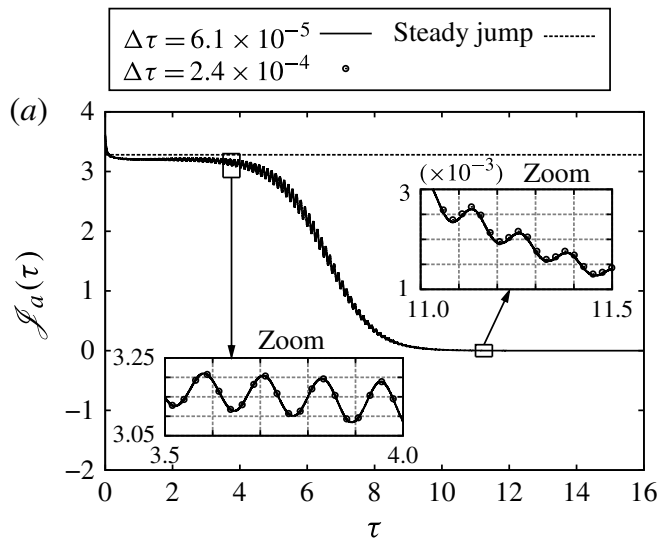

(b)

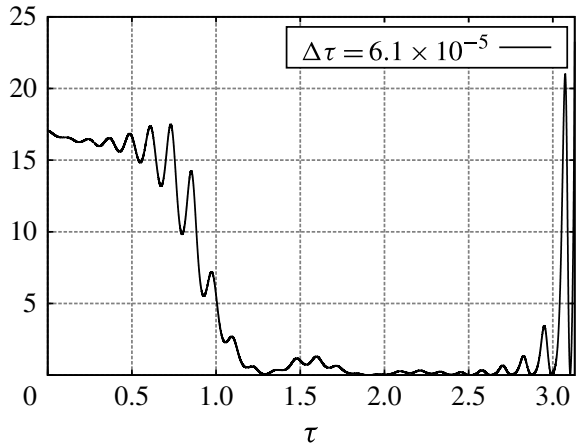

FIGURE 12. Evolution of the acoustic jump $\mathscr{J}_{a}(\tau)$ for $(a) \gamma=2.1$ and $(b) \gamma=6.2$.

can survive and remain flat in a noisy environment created by its spontaneous sound. For the case of $\gamma=6.2, \mathscr{J}_{a}$ decreases to a low level as the flame flattens, but it then amplifies very rapidly. Note that a comparison of the results for two different time steps indicates that the resolution is adequate (see figure 12a). Figure 13 shows the variation of the saturation level of the acoustic pressure when $\gamma$ varies between 2.1 (at which the first curved steady flame starts to appear) and 5.5 (when the secondary parametric instability starts occurring). The plateau level increases with moderate $\gamma$ rather rapidly, and then, interestingly, it reaches a sort of plateau. This corresponds to the threshold value above which the secondary parametric instability will be triggered.

\subsection{Theoretical confirmation}

\subsubsection{Comparison with linear stability analysis}

Using the data from the initial-value calculations shown in figure 10, it is possible to extract the growth rate of the first instability by fitting a straight line through the logarithm of the envelope of the acoustic pressure. We consider $\sigma$ as a varying parameter and measure the growth rate for different values of $\sigma$. Figure 14 shows the comparison of the extracted growth rate with the prediction by the linear stability analysis in $\$ 3.3$. For the case $\gamma=2.1$, we ran the computation for two different sets 


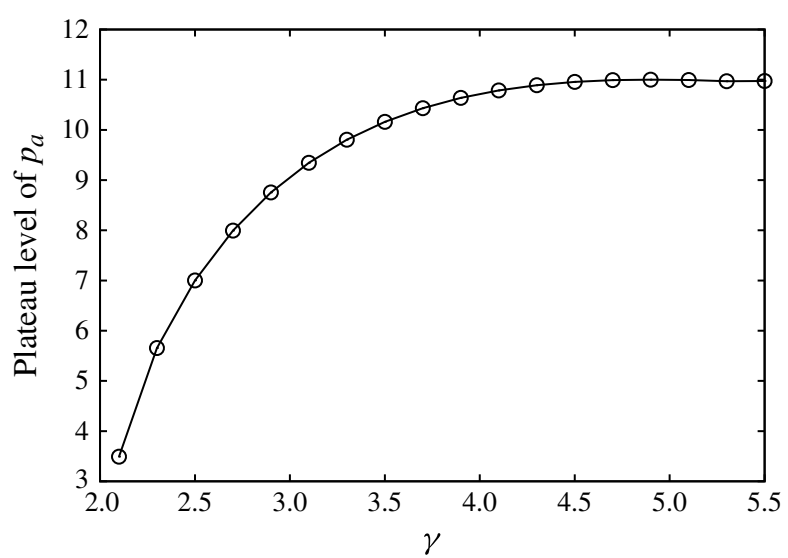

FIGURE 13. Variation of the saturation (plateau) level of the acoustic pressure with $\gamma$.
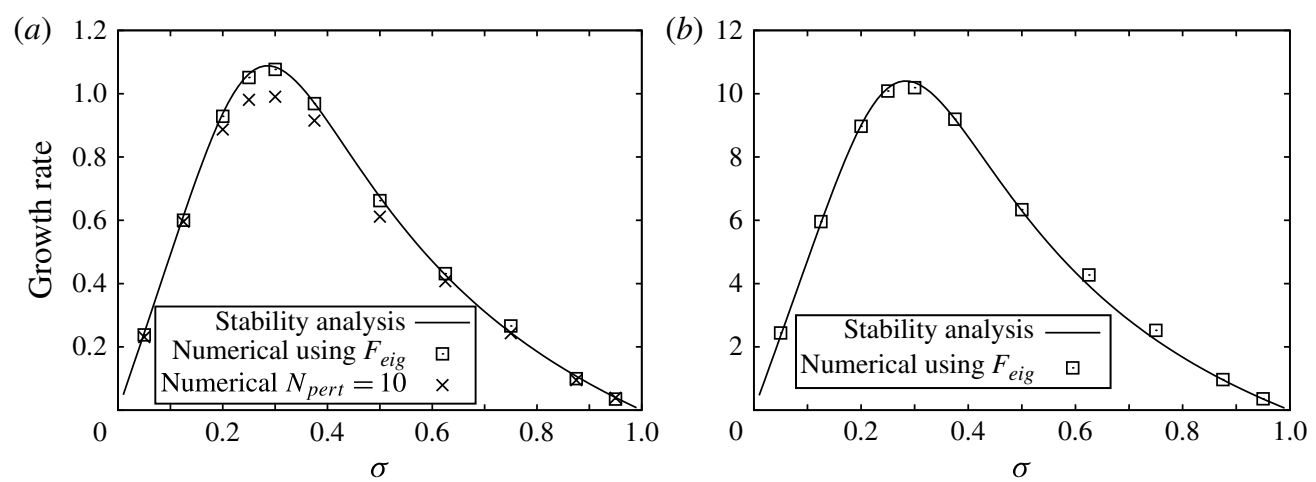

FIGURE 14. Comparison of the growth rates predicted by stability analysis and initialvalue calculations for $(a) \gamma=2.1$ and $(b) \gamma=6.2$.

of initial conditions: one given by $F_{0}=F^{S}+\epsilon \cos \left(N_{\text {pert }} \eta\right)$, and the other given by $F_{0}=F^{S}+\epsilon F_{\text {eig }}$ (see (4.4)). In the case $\gamma=6.2$, only the latter type of initial condition was used. In both cases, there is an excellent agreement between the predictions by the eigenvalue and initial-value approaches. This is a good validation for the code, and also confirms that the first observed growth corresponds to the linear instability of the steady flame.

\subsubsection{Parametric instability}

In this subsection, we aim to explain mathematically why in the case $\gamma=2.1$ the flame remains flat in a noisy environment despite being unstable in the absence of acoustic field, whereas in the case $\gamma=6.2$ a violent secondary instability occurs. The answer lies in the impact on the flame of the acoustic field, which was generated by the perturbation to the flame at an earlier stage. Figure 11 suggests that, in an established noisy environment, $\mathscr{B}_{a}(\tau)$ is nearly periodic, so that we can write

$$
\mathscr{B}_{a}(\tau)=A_{a} \cos \left(\omega_{1} \tau\right),
$$

where $\omega_{1}$ is the first acoustic mode of the duct. Furthermore, in the saturated regime, $\mathscr{J}_{a} \approx 0$, implying that the flame is no longer acting on the acoustics, and therefore 

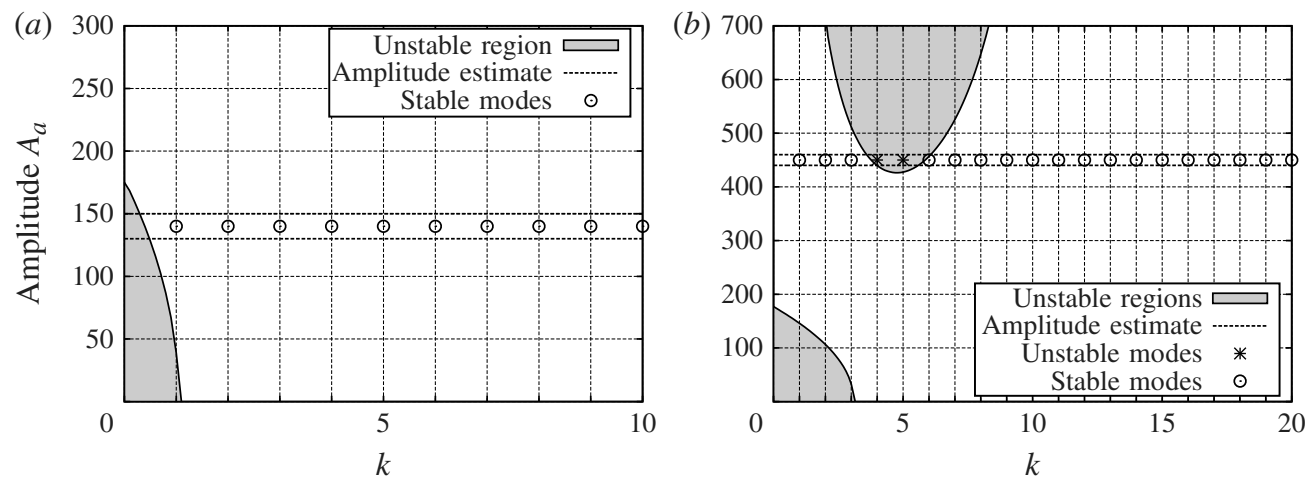

FIGURE 15. Stability boundary of (5.2) for $(a) \gamma=2.1$ and $(b) \gamma=6.2$.

we may take $A_{a}$ as a constant. On the other hand, since the flame is nearly flat, it is reasonable to linearise the spectral flame-front equation (4.1) around the flat profile and obtain

$$
A \frac{\partial^{2} \widehat{F}}{\partial \tau^{2}}(k, \tau)+B(k) \frac{\partial \widehat{F}}{\partial \tau}(k, \tau)+\left[|k| A_{a} \cos \left(\omega_{1} \tau\right)-q k^{2}+2 \delta M_{a}|k|^{3}\right] \widehat{F}(k, \tau)=0,
$$

which corresponds to the so-called damped Mathieu equation, a particular case of linear equations with periodic coefficients of period $T_{a}=2 \pi / \omega_{1}$. In order to stay close to the notation used in the literature, let $x_{1}(\tau)=\widehat{F}(k, \tau)$ and $x_{2}(\tau)=\partial \widehat{F}(k, \tau) / \partial \tau$. Then the linearised 'noisy' flame-front equation (5.2) can be rewritten as a first-order system for the vector $\boldsymbol{x}(\tau)=\left[x_{1}(\tau), x_{2}(\tau)\right]^{\mathrm{T}}$,

$$
\dot{\boldsymbol{x}}(\tau)=\mathbb{A}(\tau) \boldsymbol{x}(\tau),
$$

where $\mathbb{A}(\tau)$ is a $T_{a}$-periodic $2 \times 2$ matrix. The parametric instability can be studied using Floquet theory. Let us consider two linearly independent solutions $\boldsymbol{x}_{1}(\tau)$ and $\boldsymbol{x}_{2}(\tau)$ such that $\boldsymbol{x}_{1}(0)=[1,0]^{\mathrm{T}}$ and $\boldsymbol{x}_{2}(0)=[0,1]^{\mathrm{T}}$, and construct the $2 \times 2$ principal fundamental solution matrix $\boldsymbol{X}(\tau)=\left(\boldsymbol{x}_{1}(\tau), \boldsymbol{x}_{2}(\tau)\right)$. Integrating (5.3) from $\tau=0$ to $\tau=$ $T_{a}$, we obtain $\boldsymbol{X}\left(T_{a}\right)$. The stability of $\boldsymbol{x} \equiv 0$ (representing a flat flame) is determined by the eigenvalues of $\boldsymbol{X}\left(T_{a}\right)$, say $\rho_{1}$ and $\rho_{2}$. The system is stable if $\left|\rho_{1}\right|<1$ and $\left|\rho_{2}\right|<1$, and unstable if $\left|\rho_{1}\right|>1$ or $\left|\rho_{2}\right|>1$.

The parametric stability analysis is similar to those done by Markstein \& Squire (1955) and Searby \& Rochwerger (1991) for an externally imposed acoustic wave, but in order to interpret and substantiate our numerical results, the stability will be characterised in terms of $A_{a}$ for a given $\omega_{1}$ rather than in terms a 'reduced acoustic amplitude', and the stability boundary will be mapped out in the $A_{a}-k$ plane. Let us assume that $k$ and $\delta M_{a}$ are fixed parameters. The procedure consists of altering the amplitude $A_{a}$ to determine the values $\left(A_{a}\right)_{n}$ for marginal stability, i.e. for one of the eigenvalues to lie on the unit circle in the complex $\rho$ plane. We then repeat the procedure for different values of $k$ and obtain the stability boundary as shown in figure 15. Note that, for flames within a duct, the only relevant values of $k$ are integers. For $\gamma=2.1$, we notice that, in the vicinity of the approximated amplitude $A_{a} \approx$ 130-150 during the saturated phase, all the integer values of $k$ are in the stable region, that is, a flame is stabilised by the noise generated at an earlier stage. This is why a flat flame can be sustained in a noisy environment in this case. However, for $\gamma=6.2$ 

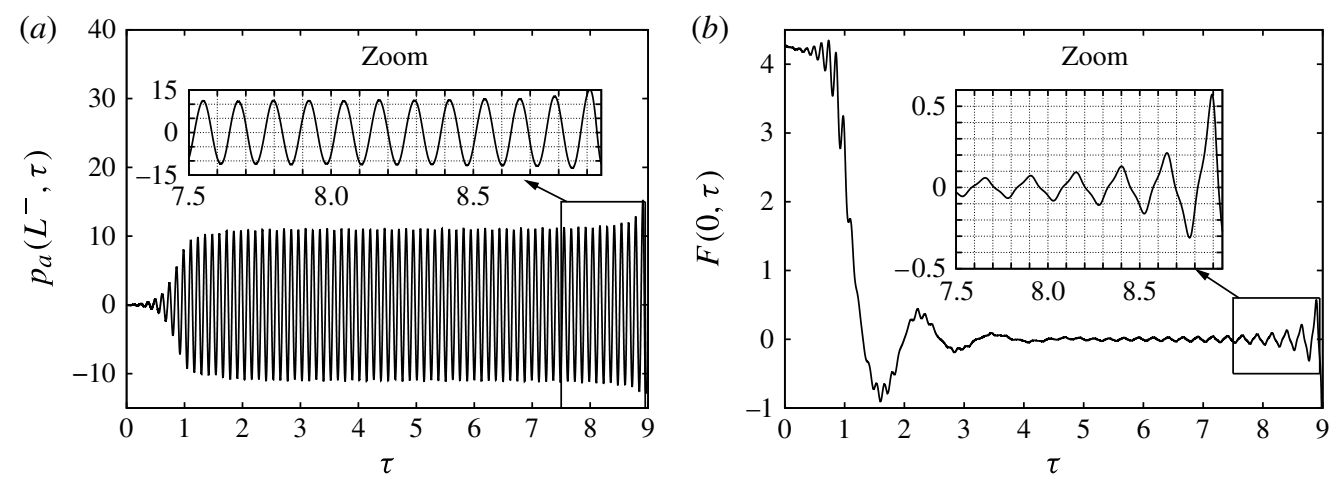

FIgURE 16. (a) Acoustic pressure at the inlet of the tube and (b) evolution of the flame at the centre of the duct for $\gamma=5.6$.

the integers $k=4$ and $k=5$ are in the unstable region for the estimated amplitude $A_{a}$, which is consistent with the fact that the wrinkling observed in figure $9(b)$ seems to have a wavenumber equal to 4 . The stability analysis shows that the upper unstable region corresponds to a subharmonic parametric instability because, on the boundary, $\rho_{1}=-1$. This means that the flame front $F$ should oscillate at the frequency $\omega_{1} / 2$ while the secondary instability is occurring. This fact gives us yet an additional way to validate our numerical solutions to the initial-value problem. In the case of $\gamma=6.2$, the time between the start of the secondary instability and the blow-up is too short to illustrate this behaviour. So instead, we have run the computation for a very similar case, $\gamma=5.6$, which exhibits a slightly smaller secondary growth rate, enabling us to observe the growth during a few acoustic cycles. In figure 16, we plot the time evolution of $(a)$ the acoustic pressure and $(b)$ the flame at the duct centre (i.e. $F(\eta=$ $0, \tau))$. A window corresponding to the secondary growth is selected, in which both signals are plotted. The frequency of flame-front oscillations is found to be half of that of the acoustic pressure, confirming that a subharmonic parametric instability of Floquet type indeed takes place.

\subsection{Comparison with experiments: the propagating flame}

An interesting experiment concerning acoustic-flame interaction and the resulting parametric instabilities was conducted by Searby (1992), where the premixed fuel was a lean propane-air mixture. Similar behaviour to those described above has been observed. However, in order to mimic as closely as possible the experimental conditions, we need to alter our computations slightly. First of all, in the experiment, the flame is propagating freely in a long tube, and hence the mean position of the flame $\sigma$ is actually changing in time. It is possible to incorporate this feature in our model by computing $\sigma$ at each time step by solving the differential equation (by AB4 again),

$$
\frac{\partial \sigma}{\partial \tau}=-\frac{h^{*}}{4 \pi \ell^{*}} \overline{(\tilde{\nabla} F)^{2}}=-\frac{h^{*}}{4 \pi \ell^{*}}\left(\mathrm{i} k^{\prime} \widehat{F}\left(k^{\prime}, \tau\right)\right) \star\left(\mathrm{i} k^{\prime} \widehat{F}\left(k^{\prime}, \tau\right)\right)(0, \tau) .
$$

Secondly, in Searby's experiment, the tube is vertical and it is necessary to include gravity, which was neglected thus far in order to compare some of our results with analytical solutions of the MS equation. We computed the steady states for different 

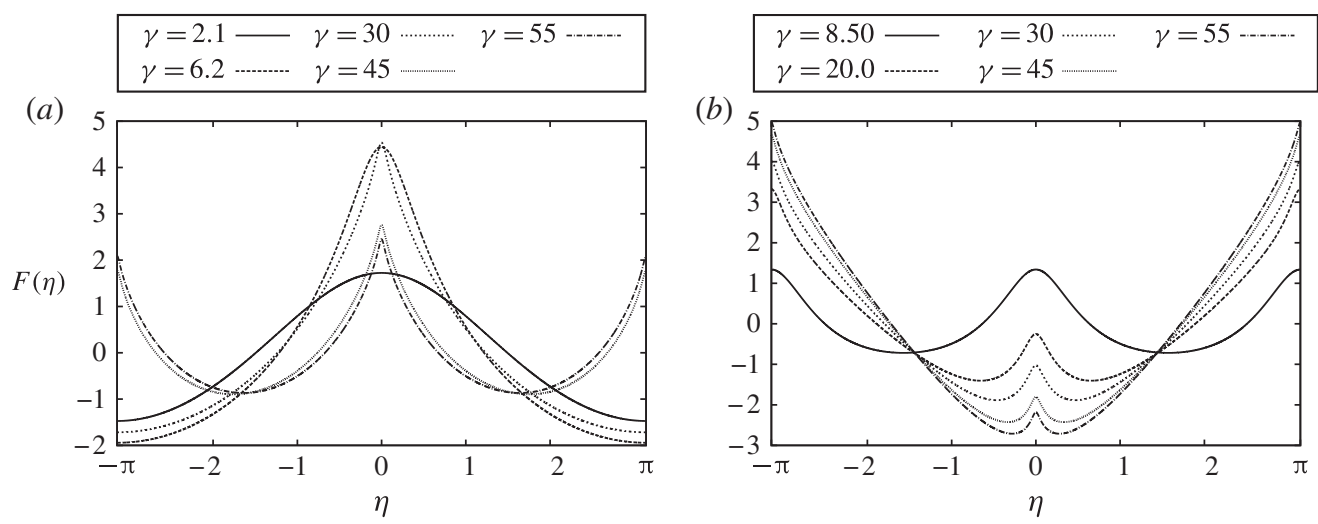

FIGURE 17. Flame shapes of the steady states for different values of $\gamma:(a)$ without gravity and $(b)$ for $G=3.14$.

$\begin{array}{lccccccc}\text { Constants } & \begin{array}{c}\Theta_{-\infty} \\ (\mathrm{K})\end{array} & \begin{array}{c}D_{t h} \\ \left(\mathrm{~cm}^{2} \mathrm{~s}^{-1}\right)\end{array} & M_{a} & \begin{array}{c}a^{*} \\ \left(\mathrm{~m} \mathrm{~s}^{-1}\right)\end{array} & \begin{array}{c}g \\ \left(\mathrm{~cm} \mathrm{~s}^{-2}\right)\end{array} & \begin{array}{c}\ell^{*} \\ (\mathrm{~m})\end{array} & \begin{array}{c}h^{*} \\ (\mathrm{~m})\end{array} \\ \text { Values } & 298 & 0.21 & 4.5 & 343 & 981 & 1.2 & 0.1\end{array}$

TABLE 3. Constants pertaining to the experiment of Searby (1992).

\begin{tabular}{lccccccccc} 
Parameters & \multicolumn{1}{c}{$\phi$} & $\begin{array}{c}\Theta_{\infty} \\
(\mathrm{K})\end{array}$ & $\Theta_{\infty} / \Theta_{-\infty}$ & $q$ & $\begin{array}{c}U_{L} \\
\left(\mathrm{~cm} \mathrm{~s}^{-1}\right)\end{array}$ & $\delta$ & $\gamma$ & $M$ & $G$ \\
Experiment 1 & 0.702 & 1886 & 6.33 & 5.33 & 22.3 & 0.0059 & 200.75 & $6.5 \times 10^{-4}$ & 3.14 \\
Experiment 2 & 0.77 & 2005 & 6.73 & 5.73 & 27.5 & 0.0048 & 265.28 & $8.0 \times 10^{-4}$ & 2.06
\end{tabular}

TABLE 4. Parameters corresponding to experiments 1 and 2, respectively.

values of $\gamma$ with or without gravity, and they are shown in figure 17. Gravity seems to have an appreciable effect on the shape of the steady flames. Thirdly, we have also assumed that the main parameters, namely $M, \gamma$ and $q$, were independent of each other, while in reality they are related. Hence, in order to mimic the experiments, we need to choose these parameters carefully. Based on the information given in Searby (1992) and Clanet et al. (1999), it is possible to recover the values of most parameters, which are given in table 3. In Searby (1992), four different experimental results (corresponding to four different values of the equivalence ratio $\phi$ ) are presented, but, as far as our study is concerned, two of them (henceforth referred to as experiments 1 and 2) have greater interest. The conditions in which these two experiments have been carried out are displayed in table 4. Most parameters are in the range of what has been used previously, but $\gamma$ is much larger, corresponding to an extremely thin flame. Numerically, it becomes challenging to compute the solutions for such a high value of $\gamma$.

The experimental results, reproduced in figure 18(a), suggest that, in the case of experiment 1, the flame, originally curved, flattens and remains flat in a noisy environment, while the acoustic pressure saturates after the first acoustic instability. Using our model and choosing all the parameters equal to those presented in tables 3 and 4 , except that $\gamma=8.5$, we obtained the numerical results presented in figure $18(b)$. 

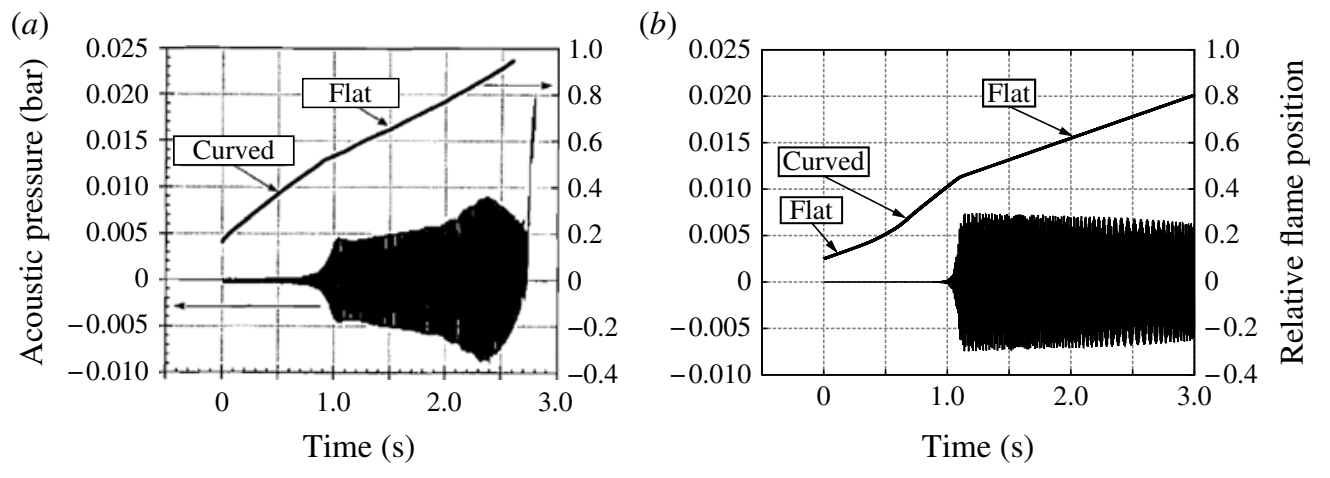

FIGURE 18. Evolution of the acoustic pressure and the relative flame position $1-\sigma$ : (a) experiment 1 taken from Searby (1992); (b) theoretical prediction for $\gamma=8.5$.
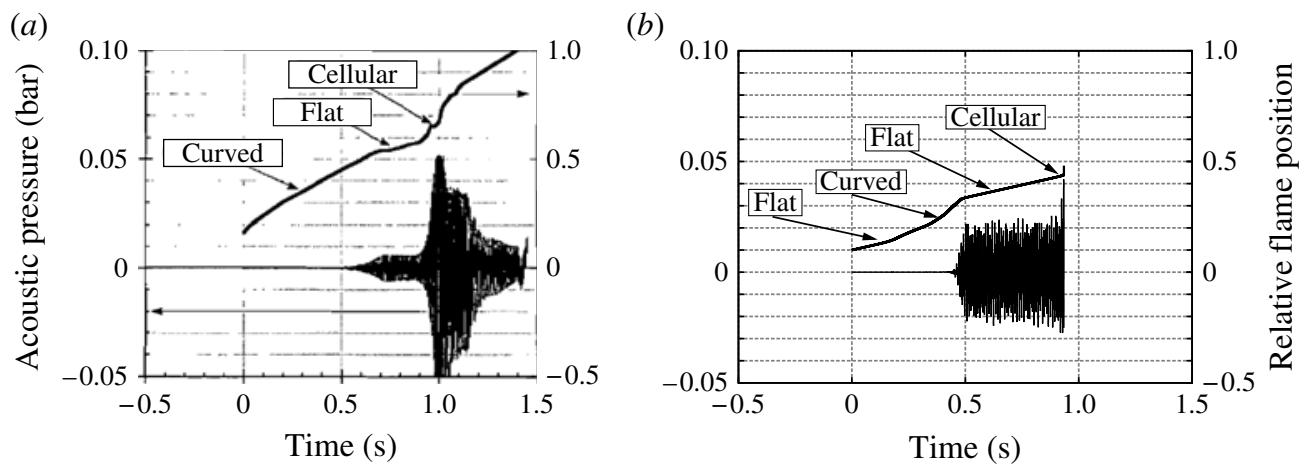

FIGURE 19. Evolution of the acoustic pressure and the relative flame position $1-\sigma$ : (a) experiment 2 taken from Searby (1992); $(b)$ theoretical prediction for $\gamma=15.441$.

Moreover, because the initial shape of the flame in the experiment is not described precisely, we started with a quasi-flat initial flame, which explains the slightly different initial behaviour of $\sigma$. As is illustrated in figure 20(a), the theory predicts that, starting from a flat initial shape, the flame tends to become curved with a shape resembling the steady state (with gravity). Indeed, the flame shape at $\tau=0.61$ is very similar to the steady state for $\gamma=8.5$ plotted in figure 17. It then starts to flatten and remains almost totally flat until the end of the tube is reached. As illustrated in figure $18(b)$, the acoustic pressure amplifies when the flame is curved. Once it reaches a certain level, it renders the flame flat and saturates as a result. These theoretical predictions are in qualitative agreement with the experimental observations.

In the case of experiment 2, as is shown in figure 19(a), the flame, originally curved, tends to flatten and remains flat for a little while. It then starts to wrinkle and eventually becomes cellular. At this stage, a secondary exponential growth of the acoustic pressure can be observed. Our model can capture such behaviour up to the sharp rise in pressure, which is much faster than the first amplification occurring in the earlier phase. Indeed, while keeping all the other parameters equal to those presented in tables 3 and 4 for experiment 2, we found that, for a value of $\gamma=15.441$, our results, also presented in figure 19(b), are comparable with the experimental observations reproduced in figure 19(a). Figure 20(b) shows that, with 

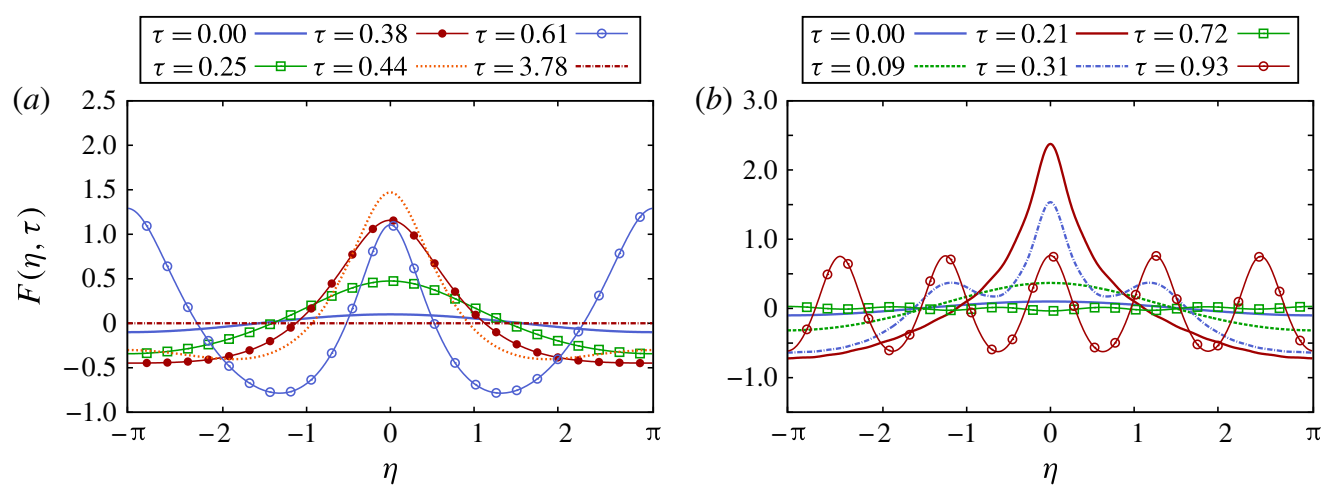

FIGURE 20. (Colour online) Evolution of the flame shape: $(a)$ theoretical prediction of experiment 1 for $\gamma=8.5$; $(b)$ theoretical prediction of experiment 2 for $\gamma=15.441$.

a prescribed initial flat state, the flame goes through a curved transient state. After this, the overall shape of the flame flattens while small wrinkles start to develop. Subsequently, the amplitude of the wrinkles starts growing exponentially towards a strongly nonlinear regime.

The quantitative differences between the experimental and numerical results may be due to the following reasons:

(i) The experiments were conducted for a fully three-dimensional case in a cylindrical tube, while our computations were performed for a rectangular duct, and we made a two-dimensional approximation.

(ii) No acoustic losses have been taken into account in our model, but in reality there must be energy dissipation associated with the tube wall.

(iii) The initial conditions in the experiments are those of an already curved flame, while we start from an almost flat shape.

(iv) The term $\boldsymbol{V} \cdot \nabla F$ has been neglected in the flame-front equation (2.20).

(v) Finally, and perhaps most importantly, a weakly nonlinear approximation is made in our model, which is the reason why it cannot predict what happens once the flame has become highly cellular in experiment 2 .

\section{Concluding remarks}

As an effort to shed further light on the fundamental mechanisms of combustion instability, a flame-flow-acoustic interaction model was derived from the asymptotic theory of WWMP by making a weak nonlinearity assumption, under which the hydrodynamic field is linearised while the geometric nonlinearity is retained. This assumption is asymptotically consistent in the limit of small heat release, but that is not the case for the $O(1)$ heat release of practical interest. The model accounts for the DL and RT instabilities. It describes the generation of the spontaneous acoustic field by the flame and its two-way interaction with the flame. Most crucially, as the flame and the spontaneous sound are both allowed to evolve in this model, the two crucial mechanisms, the parametric instability and the radiation of spontaneous sound when a flame wrinkles, operate simultaneously and are coupled dynamically as they are in experiments.

Using the model and numerical methods developed in this paper, we investigated the linear and weakly nonlinear instability of curved flames. A crucial difference from 
previous work, except that of Pelcé \& Rochwerger (1992), is that the spontaneous acoustic field, emitted by a perturbed flame, is taken into account in our study. The linear stability analysis shows that curved flames that are stable when the acoustics is ignored may actually be unstable, and this conclusion is confirmed by numerical solutions to the initial-value problem governing the flame evolution. On the other hand, we found that, when an intrinsically unstable flat flame is perturbed, it may be stabilised by the spontaneous sound. The most significant result is that the dynamic coupling of DL instability and the spontaneous acoustics may induce a secondary subharmonic parametric instability. These results emphasise the importance of considering both acoustic-flame interactions and flow-flame interactions. Indeed, purely hydrodynamic models such as the MS equation cannot predict the primary instability for the particular steady curved flames considered in this paper, and purely kinematic models such as the $G$ equation (coupled with the acoustic field) cannot predict the secondary instability because the latter is caused by the dynamic RT effect. Our model is able to reproduce, at least qualitatively, the primary and secondary instabilities observed experimentally in Searby (1992). Even though the primary and secondary instabilities have been previously observed and studied, such a sequence of instabilities, to our knowledge, has not been predicted by any other models.

The model proposed in the present work retains all the key physical factors of combustion instability, but its main limitation is the weak nonlinearity assumption. In order to take strong nonlinearity into account, it is necessary to develop an efficient numerical method for solving the full system governing flame-flow-acoustic interactions. In this paper, we have considered only freely propagating flames, for which the action of the sound on the flame is solely through the unsteady RT effects associated with the acoustic acceleration. For anchored flames, the RT mechanism and the kinematic advection by the acoustic velocity both operate, but the former has not been considered in existing models where the $G$ equation is used to describe the flame motion. It would be interesting to develop an improved model in which both mechanisms are included and their relative importance can be assessed. These are problems to be tackled in the next phase of our investigation.

\section{Acknowledgements}

The authors would like to acknowledge the support by UK EPSRC (EP/I017240/1). The referees are thanked for their constructive comments and suggestions, which have helped improve the present paper.

\section{Appendix A. Steady-state solutions of the flame equation}

In this appendix, we consider steady solutions to the problem described in § 2.3. For simplicity, the flow is assumed to be two-dimensional, and so the space variables are reduced from $(\xi, \eta, \zeta)$ to $(\xi, \eta)$, and the vector variable $\boldsymbol{V}$ becomes the scalar variable $V$. With $\partial U / \partial \tau=\partial V / \partial \tau=0$, the equations (2.31) simplify to

$$
\frac{\partial U}{\partial \xi}=-\frac{\partial V}{\partial \eta}, \quad \frac{\partial U}{\partial \xi}=-\frac{\partial P}{\partial \xi}, \quad \frac{\partial V}{\partial \xi}=-\frac{\partial P}{\partial \eta} .
$$

Since acoustic fluctuations are absent $\left(\mathscr{B}_{a}(\tau)=0\right)$, the jump conditions $(2.32)$ become

$$
[U]_{-}^{+}=0, \quad[V]_{-}^{+}=-q \frac{\partial F}{\partial \eta}, \quad[P]_{-}^{+}=-\frac{q G}{1+q} F .
$$


As was mentioned in $\S 2.2$, for steady states, $\partial F / \partial \tau=U_{F}$, with $U_{F}$ being the propagation speed of the flame. However, in order to obtain steady solutions by time marching numerically, we shall retain the term $\partial F / \partial \tau$ in the flame equation (2.33), which becomes

$$
\frac{\partial F}{\partial \tau}=U\left(0^{-}, \eta\right)-\frac{1}{2}\left(\frac{\partial F}{\partial \eta}\right)^{2}+\delta M_{a} \frac{\partial^{2} F}{\partial \eta^{2}} .
$$

The system (A 1)-(A 3) can be reduced to a single equation by Fourier analysis. Let the Fourier transform with respect to $\eta$ of any function $\phi(\xi, \eta, \tau)$ be denoted $\widehat{\phi}(\xi, k, \tau)$. Taking the Fourier transform of (A 1) and solving the resultant equations, we obtain

$$
(\widehat{P}, \widehat{U}, \widehat{V})=\left(\phi_{ \pm} \mathrm{e}^{\mp|k| \xi},-\phi_{ \pm} \mathrm{e}^{\mp|k| \xi}+C_{ \pm}(k), \pm \mathrm{i} \frac{k}{|k|} \phi_{ \pm} \mathrm{e}^{\mp|k| \xi}\right)
$$

where the \pm signs refer to $\xi>0$ and $\xi<0$, respectively, and $\phi_{ \pm}$and $C_{ \pm}$are unknown functions of $k$ only. Since we assume that no vortical disturbance is present upstream of the flame, $C_{-}=0$. Using the Fourier transform of the jump conditions (A 2), we find that

$$
\widehat{U}\left(0^{-}, k\right)=-\phi_{-}=\frac{q \widehat{F}}{2}\left(|k|-\frac{G}{1+q}\right) .
$$

Inversion of $\widehat{U}\left(0^{-}, k\right)$ gives $U\left(0^{-}, \eta\right)$. By using this, the flame equation (A 3) is written, in physical space, as

$$
\left.\begin{array}{l}
\frac{\partial F}{\partial \tau}=U\left(0^{-}, \eta\right)-\frac{1}{2}\left(\frac{\partial F}{\partial \eta}\right)^{2}+\delta M_{a} \frac{\partial^{2} F}{\partial \eta^{2}}, \\
\widehat{U}\left(0^{-}, k\right)=\frac{1}{2} q\left(|k|-\frac{G}{1+q}\right) \widehat{F},
\end{array}\right\}
$$

which is equivalent to the well-known MS equation when $G=0$.

Combining (A 5) with the Fourier transform of the flame equation (A 3), we obtain a single equation in spectral space:

$$
\frac{\partial \widehat{F}}{\partial \tau}=\frac{q}{2}\left(|k|-\frac{G}{1+q}\right) \widehat{F}-\frac{1}{2}\left(\mathrm{i} k^{\prime} \widehat{F}\left(k^{\prime}\right)\right) \star\left(\mathrm{i} k^{\prime} \widehat{F}\left(k^{\prime}\right)\right)(k)-\delta M_{a} k^{2} \widehat{F},
$$

where $\star$ denotes a convolution, with $k^{\prime}$ being a dummy variable introduced to avoid confusion. For a flame in a duct, $F$ is defined for $\eta \in[-\pi, \pi]$, and $k$ takes discrete value with $\widehat{F}(n, \tau)=\widehat{F}_{n}(\tau), \forall n \in \mathbb{Z}$. In the discrete form, the flame-front equation (A 7) reads

$$
\frac{\partial \widehat{F}_{n}}{\partial \tau}=\frac{q}{2}\left(|n|-\frac{G}{1+q}\right) \widehat{F}_{n}+\frac{1}{2} \sum_{m=-\infty}^{\infty}(n-m) m \widehat{F}_{n-m} \widehat{F}_{m}-\delta M_{a} n^{2} \widehat{F}_{n}, \quad n>0 .
$$

Here we only need to compute $\widehat{F}_{n}$ for $n>0$, since $\widehat{\phi}_{-n}=\widehat{\phi}_{n}$ for a function $\phi$ that is real and even (which is the case for $F$ ). The Fourier series is truncated by setting $\widehat{F}_{n}=0$ for any $n$ with $|n|>N$. The truncated system (A 8) consists of $N$ coupled ordinary differential equations, and is solved using a fourth-order Runge-Kutta method. The shape of the flame can be reconstructed using a truncated Fourier series. 
Appendix B. Solution to hydrodynamic equations (3.14) subject to (3.15) and the derivation of (3.19)

The hydrodynamic equations in spectral space, (3.14) subject to (3.15), are solved to obtain $\widehat{\mathfrak{P}}(\xi, k), \widehat{\mathfrak{U}}(\xi, k)$ and $\widehat{\mathfrak{V}}(\xi, k)$ :

$$
\begin{aligned}
(\widehat{\mathfrak{P}}, \widehat{\mathfrak{U}}) & =\left(\varphi_{ \pm}(k) \mathrm{e}^{\mp|k| \xi}, \mathfrak{C}_{ \pm} \mathrm{e}^{-\mathrm{i} \omega \xi R_{ \pm}}-\frac{|k| \varphi_{ \pm}}{\mathrm{i} \omega R_{ \pm} \mp|k|} \mathrm{e}^{\mp|k| \xi}\right), \\
\widehat{\mathfrak{V}} & =\mathfrak{D}_{ \pm} \mathrm{e}^{-\mathrm{i} \omega \xi R_{ \pm}}-\frac{\mathrm{i} k \varphi_{ \pm}}{\mathrm{i} \omega R_{ \pm} \mp|k|} \mathrm{e}^{\mp|k| \xi}
\end{aligned}
$$

where the \pm signs refer to $\xi>0$ and $\xi<0$, respectively, and $\varphi_{ \pm}, \mathfrak{C}_{ \pm}$and $\mathfrak{D}_{ \pm}$are unknown functions of $k$. The continuity equation in the frequency space and the absence of vortical disturbances in the oncoming mixture imply, respectively, that

$$
-\mathrm{i} \omega R_{ \pm} \mathfrak{C}_{ \pm}+\mathrm{i} k \mathfrak{D}_{ \pm}=0 \text { and } \mathrm{i} k \mathfrak{C}_{-}+\mathrm{i} \omega R_{-} \mathfrak{D}_{-}=0
$$

which leads to $\mathfrak{C}_{-}=\mathfrak{D}_{-}=0$. Taking the Fourier transform of the three jump conditions (3.15) in the frequency space and using (B 1) and (B 2), we obtain a linear system of three equations for as many unknowns (namely $\varphi_{ \pm}$and $\mathfrak{C}_{+}$). Solving this system, we find

$$
\varphi_{-}=\frac{|k|+\mathrm{i} \omega R_{-}}{\left(\mathrm{i} \omega R_{+}+|k|\right)+\left(|k|+\mathrm{i} \omega R_{-}\right)}\left\{q\left(\frac{G}{1+q}-|k|\right) \widehat{\mathfrak{F}}+\mathfrak{B}_{\mathfrak{a}} \widehat{F}^{S}\right\} .
$$

Hence we find a closed form of the spectral flame equation in the frequency space,

$$
\mathrm{i} \omega \widehat{\mathfrak{F}}=\frac{-|k|\left\{q\left(\frac{G}{1+q}-|k|\right) \widehat{\mathfrak{F}}+\mathfrak{B}_{\mathfrak{a}} \widehat{F}^{S}\right\}}{\left(\mathrm{i} \omega R_{+}+|k|\right)+\left(|k|+\mathrm{i} \omega R_{-}\right)}-\left(\mathrm{i} k^{\prime} \widehat{F}^{S}\left(k^{\prime}\right)\right) \star\left(\mathrm{i} k^{\prime} \widehat{\mathfrak{F}}\left(k^{\prime}\right)\right)(k)-\delta M_{a} k^{2} \widehat{\mathfrak{F}},
$$

where $\mathfrak{B}_{\mathfrak{a}}$ is given by (3.18). Alternatively, the above system can be written as

$$
\left.\begin{array}{l}
\frac{\partial \tilde{F}}{\partial \tau}=\tilde{U}\left(0^{-}, \eta\right)-\frac{\partial F^{S}}{\partial \eta} \frac{\partial \tilde{F}}{\partial \eta}+\delta M_{a} \frac{\partial^{2} \tilde{F}}{\partial \eta^{2}}, \\
\widehat{\mathfrak{U}}\left(0^{-}, k\right)=\frac{-|k|\left\{q\left(\frac{G}{1+q}-|k|\right) \widehat{\mathfrak{F}}+\mathfrak{B}_{\mathfrak{a}} \widehat{F}^{S}\right\}}{\left(\mathrm{i} \omega R_{+}+|k|\right)+\left(|k|+\mathrm{i} \omega R_{-}\right)} .
\end{array}\right\}
$$

Note that, if the time derivative of the perturbed hydrodynamic quantities is neglected, the terms $\mathrm{i} \omega R_{ \pm}$disappear in (B 6). Moreover, if acoustic fluctuations are artificially suppressed, then $\mathfrak{B}_{\mathfrak{a}}=0$ in (B 6). If, finally, gravity is also neglected, then (B 6) reduces to the linearised perturbed MS equation (3.4) via the changes of variable $t=q \tau, \psi=-\tilde{F} / q$ and $\delta M_{a}=q / \gamma$. Substitution of (3.18) into (B 5) yields (3.19).

\section{Appendix C. Derivation of the spectral flame equation (4.1)}

The aim of this appendix is to reduce the system (2.31)-(2.33) in spectral space to a single equation governing the flame front. In a similar manner as in appendices A and $\mathrm{B}$, equations (2.31) are Fourier-transformed with respect to $\eta$ and $\zeta$, and the 
resulting equations can be manipulated to obtain $\partial^{2} \widehat{P} / \partial \xi^{2}-k^{2} \widehat{P}=0$, where $k$ is such that $k^{2}=k_{2}^{2}+k_{3}^{2}$. The general solution can be written as (see Wu \& Law 2009)

$$
\begin{aligned}
& \widehat{U}(\xi, k, \tau)=\phi_{ \pm}(k, \tau) \mathrm{e}^{\mp|k| \xi}+C_{ \pm}\left(\tau-R_{ \pm} \xi\right), \\
& \widehat{P}(\xi, k, \tau)= \pm|k|^{-1}\left(R_{ \pm} \phi_{ \pm}^{\prime}(\tau) \mp|k| \phi_{ \pm}(\tau)\right) \mathrm{e}^{\mp|k| \xi}, \\
& \widehat{\boldsymbol{V}}(\xi, k, \tau)=\mp \mathrm{i}(\boldsymbol{k} /|k|) \phi_{ \pm}(\tau) \mathrm{e}^{\mp|k| \xi}+\boldsymbol{D}_{ \pm}\left(\tau-R_{-} \xi\right),
\end{aligned}
$$

where the \pm signs refer to $\xi>0$ and $\xi<0$, respectively, $\phi_{ \pm}$and $C_{ \pm}$are unknown functions, $\boldsymbol{k}=\left(k_{2}, k_{3}\right)^{\mathrm{T}}$ and $\boldsymbol{D}_{ \pm}$are unknown vector functions. Since no vortical disturbances are present in the fresh mixture, it can be shown that $C_{-}=\boldsymbol{D}_{-}=0$ as was done in appendix B. The continuity equation in (2.31) implies that

$$
-R_{+} C_{+}^{\prime}(\tau)+\mathrm{i} \boldsymbol{k} \cdot \boldsymbol{D}_{+}(\tau)=0 .
$$

The partially linearised flame-front equation (2.33) is Fourier-transformed to

$$
\frac{\partial \widehat{F}}{\partial \tau}(k, \tau)=\phi_{-}(\tau)-\frac{1}{2}(\mathrm{i} k \widehat{F}) \star(\mathrm{i} k \widehat{F})-\delta M_{a} k^{2} \widehat{F}(k, \tau),
$$

where (C 1) has been used. By differentiating (C 5) with respect to $\tau$, we obtain

$$
\phi_{-}^{\prime}(\tau)=\frac{\partial^{2} \widehat{F}}{\partial \tau^{2}}(k, \tau)+(\mathrm{i} k \widehat{F}) \star\left(\mathrm{i} k \frac{\partial \widehat{F}}{\partial \tau}\right)+\delta M_{a} k^{2} \frac{\partial \widehat{F}}{\partial \tau}(k, \tau) .
$$

In order to determine the unknown functions in the expressions of $\widehat{U}, \widehat{V}$ and $\widehat{P}$, let us use the hydrodynamic jump conditions (2.32), which are Fourier-transformed to

$$
[\widehat{U}]_{-}^{+}=0, \quad[\widehat{V}]_{-}^{+}=-\mathrm{i} q \boldsymbol{k} \widehat{F}, \quad[\widehat{P}]_{-}^{+}=-\left[\mathscr{B}_{a}(\tau)+q G /(1+q)\right] \widehat{F} . \quad(\mathrm{C} 7 a-c)
$$

Substitution of (C 1)-(C 3) into (C 7) gives three equations, from which we find that

$$
\begin{aligned}
\left(R_{+}+R_{-}\right) \phi_{-}^{\prime}(\tau)-\left(R_{+} C_{+}^{\prime}-|k| C_{+}\right) & =-|k|\left[\mathscr{B}_{a}(\tau)+q G /(1+q)\right] \widehat{F}(k, \tau), \\
\left(\mathrm{i} k \cdot D_{+}-|k| C_{+}\right) & =q k^{2} \widehat{F}-2|k| \phi_{-} .
\end{aligned}
$$

Use of (C 4) and (C9) in (C 8) to eliminate $C_{+}, C_{+}^{\prime}$ and $\boldsymbol{D}_{+}$gives

$$
\left(R_{+}+R_{-}\right) \phi_{-}^{\prime}(\tau)=\mathrm{i} \boldsymbol{k} \cdot \boldsymbol{D}_{+}-|k| C_{+}-|k|\left[\mathscr{B}_{a}(\tau)+q G /(1+q)\right] \widehat{F}(k, \tau) .
$$

Therefore, use of (C9) in (C 10) leads to

$$
\left(R_{+}+R_{-}\right) \phi_{-}^{\prime}(\tau)+2|k| \phi_{-}=q k^{2} \widehat{F}-|k|\left[\mathscr{B}_{a}(\tau)+q G /(1+q)\right] \widehat{F}(k, \tau) .
$$

Substituting (C 5) and (C6) into (C 11), we finally obtain

$$
A \frac{\partial^{2} \widehat{F}}{\partial \tau^{2}}+B \frac{\partial \widehat{F}}{\partial \tau}+C \widehat{F}=-|k|(\mathrm{i} k \widehat{F}) \star(\mathrm{i} k \widehat{F})-A(\mathrm{i} k \widehat{F}) \star\left(\mathrm{i} k \frac{\partial \widehat{F}}{\partial \tau}\right),
$$

where $A=R_{+}+R_{-}$,

$$
B(k)=A \delta M_{a} k^{2}+2|k|, \quad C(k, \tau)=|k|\left[\mathscr{B}_{a}(\tau)+q G /(1+q)\right]-q k^{2}+2 \delta M_{a}|k|^{3} .
$$

$(\mathrm{C} 13 a, b)$

For a two-dimensional flame, (C 12) reduces to (4.1). 


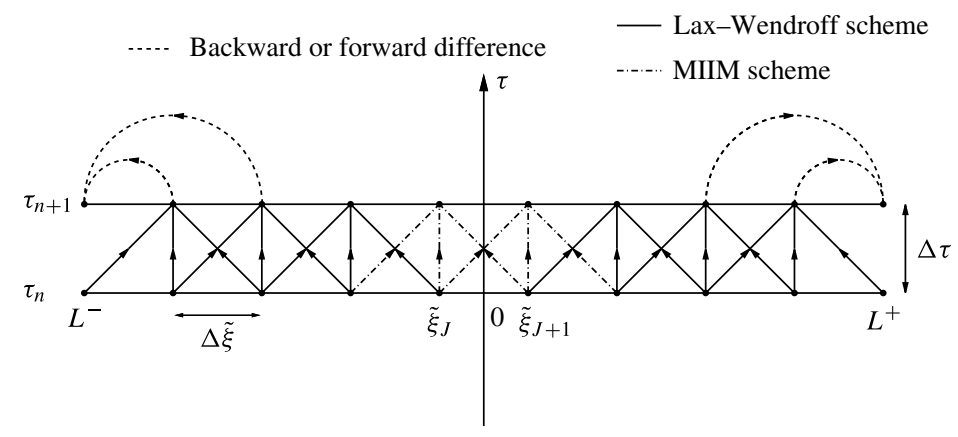

FIGURE 21. Illustration of the MIIM.

\section{Appendix D. Modified immersed interface method (MIIM)}

As an alternative to the method of characteristics, a full numerical method can be constructed to solve the acoustic system (4.5). Special attention must be paid to the discontinuities of the coefficient and the solutions. By defining the vector variable $\boldsymbol{U}=$ $\left(p_{a}, u_{a}\right)^{\mathrm{T}}$, the system (4.5) can be rewritten as a first-order hyperbolic system:

$$
\boldsymbol{U}_{\tau}+\boldsymbol{C} \boldsymbol{U}_{\tilde{\xi}}=0, \quad \text { where } \boldsymbol{C}=\left(\begin{array}{cc}
0 & 1 \\
c^{2} & 0
\end{array}\right)
$$

The jump conditions (4.7) together with (D 1) lead to higher-order jump conditions:

$$
\llbracket \boldsymbol{U} \rrbracket_{-}^{+}=\left(\begin{array}{c}
0 \\
\mathscr{J}_{a}(\tau)
\end{array}\right), \quad \llbracket \boldsymbol{C} \boldsymbol{U}_{\tilde{\xi}} \rrbracket_{-}^{+}=\left(\begin{array}{c}
0 \\
-\mathscr{J}_{a}^{\prime}(\tau)
\end{array}\right), \quad \llbracket \boldsymbol{C}^{2} \boldsymbol{U}_{\tilde{\xi} \tilde{\xi}} \rrbracket_{-}^{+}=\left(\begin{array}{c}
0 \\
\mathscr{J}_{a}^{\prime \prime}(\tau)
\end{array}\right) .
$$

For later reference, we introduce the notation $E_{1}^{+}(\tau)=E_{1}^{-}(\tau)=\llbracket \boldsymbol{U} \rrbracket_{-}^{+}$and

$$
E_{2}^{ \pm}(\tau)=\left(\boldsymbol{C}^{ \pm}\right)^{-1} \llbracket \boldsymbol{C} \boldsymbol{U}_{\tilde{\xi}} \rrbracket_{-}^{+}, \quad E_{3}^{ \pm}(\tau)=\left(\left(\boldsymbol{C}^{ \pm}\right)^{2}\right)^{-1} \llbracket \boldsymbol{C}^{2} \boldsymbol{U}_{\tilde{\xi} \tilde{\xi}} \rrbracket_{-}^{+} .
$$

In order to deal with the discontinuity of the coefficient of (4.5), the immersed interface method (Zhang \& LeVeque 1997) will be adopted and modified to take into account the jump across $\tilde{\xi}=0$. Let us consider a uniform grid, with a space mesh size $\Delta \tilde{\xi}$ and a time step $\Delta \tau$, so that the point $\tilde{\xi}=0$, where the discontinuity occurs, is not a mesh point (see figure 21). The coordinate $\tilde{\xi}$ is hence discretised by $\tilde{\xi}_{j}=L^{-}+j \Delta \tilde{\xi}$ and the time is discretised by $\tau_{n}=n \Delta \tau$. All the points that are not directly adjacent to the discontinuity shall be referred to as regular, whilst those points that are shall be referred to as irregular. Typically, for a given time, only two points are irregular, one just before the discontinuity and one just after. These points on the $\tilde{\xi}$-axis are denoted by $\tilde{\xi}_{J}$ and $\tilde{\xi}_{J+1}$, respectively (see figure 21 ). In addition, let $\boldsymbol{U}_{i}^{n}$ denote the numerical approximation of $\boldsymbol{U}\left(\tilde{\xi}_{i}, \tau_{n}\right)$. At all the regular points (apart from the extremities of the domain), one can apply the standard three-point Lax-Wendroff scheme to (D 1), leading to

$$
\boldsymbol{U}_{i}^{n+1}=\boldsymbol{U}_{i}^{n}-\frac{\Delta \tau \boldsymbol{C}_{i}}{2 \Delta \tilde{\xi}}\left(\boldsymbol{U}_{i+1}^{n}-\boldsymbol{U}_{i-1}^{n}\right)+\frac{1}{2}\left(\frac{\Delta \tau \boldsymbol{C}_{i}}{\Delta \tilde{\xi}}\right)^{2}\left(\boldsymbol{U}_{i+1}^{n}-2 \boldsymbol{U}_{i}^{n}+\boldsymbol{U}_{i-1}^{n}\right)
$$


while the extremities can be dealt with by using forward or backward difference. With this scheme, numerical stability is subject to the CFL condition,

$$
\frac{\Delta \tau}{\Delta \tilde{\xi}} \max \left(c^{+}, c^{-}\right) \leqslant 1
$$

The main premise of this method is to create artificially a three-point scheme at irregular points by writing

$$
\begin{aligned}
\boldsymbol{U}_{J}^{n+1}= & \boldsymbol{U}_{J}^{n}+\frac{\Delta \tau}{\Delta \tilde{\xi}}\left(\Gamma_{J, 1} \boldsymbol{U}_{J-1}^{n}+\Gamma_{J, 2} \boldsymbol{U}_{J}^{n}+\Gamma_{J, 3} \boldsymbol{U}_{J+1}^{n}\right) \\
& -\frac{\Delta \tau}{\Delta \tilde{\xi}} \Gamma_{J, 3} \sum_{m=0}^{2} \frac{\left(\tilde{\xi}_{J+1}\right)^{m}}{m !} E_{m+1}^{+}\left(\tau_{n}\right), \\
\boldsymbol{U}_{J+1}^{n+1}= & \boldsymbol{U}_{J+1}^{n}+\frac{\Delta \tau}{\Delta \tilde{\xi}}\left(\Gamma_{J+1,3} \boldsymbol{U}_{J}^{n}+\Gamma_{J+1,2} \boldsymbol{U}_{J+1}^{n}+\Gamma_{J+1,1} \boldsymbol{U}_{J+2}^{n}\right) \\
& +\frac{\Delta \tau}{\Delta \tilde{\xi}} \Gamma_{J+1,3} \sum_{m=0}^{2} \frac{\left(\tilde{\xi}_{J}\right)^{m}}{m !} E_{m+1}^{-}\left(\tau_{n}\right),
\end{aligned}
$$

where $E_{m}^{ \pm}$are defined in (D 3) and the $2 \times 2$ matrix coefficients $\Gamma$ can be determined analytically by enforcing a local truncation error of $O\left((\Delta \xi)^{2},(\Delta \tau)^{2}\right)$. The main difference between this modified method and the standard immersed interface method (Zhang \& LeVeque 1997) is the addition of the terms involving $E_{m}^{ \pm}$in the three-point scheme in order to deal with a function $\mathscr{J}_{a}$ that is not equal to zero, but varies with time. We note that other innovative methods such as the modified solutions presented in Piraux \& Lombard (2001) could probably be implemented as well.

\section{REFERENCES}

AldRedge, R. C. 2005 Saffman-Taylor influence on flame propagation in thermoacoustically excited flow. Combust. Sci. Technol. 177, 53-73.

Al-Shahrany, A. S., Bradley, D., Lawes, M., LiU, K. \& Woolley, R. 2006 Darrieus-Landau and thermo-acoustic instabilities in closed vessel explosions. Combust. Sci. Technol. 178, $1771-1802$.

Altantzis, C., Frouzakis, C. E., Tomboulides, A. G., Matalon, M. \& Boulouchos, K. 2012 Hydrodynamic and thermodiffusive instability effects on the evolution of laminar planar lean premixed hydrogen flames. J. Fluid Mech. 700, 329-361.

Bloxsidge, G. J., Dowling, A. P. \& LAnghorne, P. J. 1988 Reheat buzz: an acoustically coupled combustion. Part 2. Theory. J. Fluid Mech. 193, 445-473.

ByCHKOV, V. V. 1998 Nonlinear equation for a curved stationary flame and the flame velocity. Phys. Fluids 10 (8), 2091-2098.

Bychкov, V. V. 1999 Analytical scalings for flame interaction with sound waves. Phys. Fluids 11 (10), 3168-3173.

Bychkov, V. V. \& Liberman, M. A. 2000 Dynamics and stability of premixed flames. Phys. Rep. 325 (4-5), 115-237.

Cambray, P. \& Joulin, G. 1994 Length-scales of wrinkling of weakly-forced, unstable premixed flames. Combust. Sci. Technol. 97, 405-428.

CAndel, S. 2002 Combustion dynamics and control: progress and challenges. Proc. Combust. Inst. 29, 1-28. 
Clanet, C. \& Searby, G. 1998 First experimental study of the Darrieus-Landau instability. Phys. Rev. Lett. 80 (17), 3867-3870.

Clanet, C., Searby, G. \& Clavin, P. 1999 Primary acoustic instability of flames propagating in tubes: cases of spray and premixed gas combustion. J. Fluid Mech. 385, 157-197.

Clavin, P. 1985 Dynamic behavior of premixed flame fronts in laminar and turbulent flows. Prog. Energy Combust. Sci. 11, 1-59.

Clavin, P. 1994 Premixed combustion and gasdynamics. Annu. Rev. Fluid Mech. 26, 321-352.

Clavin, P. \& Graña Otero, J. C. 2011 Curved and stretched flames: the two Markstein numbers. J. Fluid Mech. 686, 187-217.

Clavin, P. \& Williams, F. A. 1982 Effects of molecular diffusion and of thermal expansion on the structure and dynamics of premixed flames in turbulent flows of large scale and low intensity. J. Fluid Mech. 116, 251-282.

Creta, F. \& Matalon, M. 2011 Propagation of wrinkled turbulent flames in the context of hydrodynamic theory. J. Fluid Mech. 680, 225-264.

DARrieus, G. 1938 Propagation d'un front de flamme. Essai de théorie des vitesses anormale de déflagration par développement spontané de la turbulence. Unpublished works presented at La Technique Moderne.

Dowling, A. P. 1995 The calculation of thermoacoustic oscillations. J. Sound Vib. 180 (4), 557-581.

Dowling, A. P. 1999 A kinematic model of a ducted flame. J. Fluid Mech. 394, 51-72.

Dowling, A. P. \& Morgans, A. S. 2005 Feedback control of combustion oscillations. Annu. Rev. Fluid Mech. 37 (1), 151-182.

Ducruix, S., Candel, S., Durox, D. \& Schuller, T. 2003 Combustion dynamics and instabilities: elementary coupling and driving mechanisms. J. Propul. Power 19 (5), 722-734.

Ducruix, S., Durox, D. \& CAndel, S. 2000 Theoretical and experimental determinations of the transfer function of a laminar premixed flame. Proc. Combust. Inst. 28, 765-773.

Durox, D., Schuller, T., Noiray, N., Birbaud, A. L. \& CAndel, S. 2009 Rayleigh criterion and acoustic energy balance in unconfined self-sustained oscillating flames. Combust. Flame 156 (1), 106-119.

Fogla, N., Creta, F. \& Matalon, M. 2013 Influence of the Darrieus-Landau instability on the propagation of planar turbulent flames. Proc. Combust. Inst. 34 (1), 1509-1517.

Gonzalez, M. 1996 Acoustic instability of a premixed flame propagating in a tube. Combust. Flame 107 (3), 245-259.

Harrje, D. T. \& Reardon, F. H. 1972 Liquid propellant rocket combustion instability. NASA Rep. SP-194.

Helenbrook, B. T. \& LAw, C. K. 1999 The role of Landau-Darrieus instability in large scale flows. Combust. Flame 117 (98), 155-169.

Kerstein, A. R., Ashurst, W. T. \& Williams, F. A. 1988 Field equation for interface propagation in an unsteady homogeneous flow field. Phys. Rev. A 37 (7), 2728-2731.

Landau, L. 1944 On the theory of slow combustion. Acta Physicochim. USSR 19, 77-85.

Lieuwen, T. 2003 Modeling premixed combustion-acoustic wave interactions: a review. J. Propul. Power 19 (5), 765-781.

LIEUWEN, T. 2005 Nonlinear kinematic response of premixed flames to harmonic velocity disturbances. Proc. Combust. Inst. 30 (2), 1725-1732.

Lieuwen, T. \& YANG, V. 2005 Combustion instabilities in gas turbine engines: operational experience, fundamental mechanisms, and modeling. Prog. Astronaut. Aeronaut. AIAA Paper 210.

Luzzato, C. M., Assier, R. C., Morgans, A. S. \& WU, X. 2013 Modelling thermo-acoustic instabilities of an anchored laminar flame in a simple lean premixed combustor: including hydrodynamic effects. In Proceedings of the 19th AIAA/CEAS Aeroacoustics Conference, 20132003, pp. 1-15.

Mallard, F. E. \& Le ChÂtelier, H. L. 1882 Étude sur la combustion des mélanges gazeux explosifs. J. Phys. Theor. Appl. 1 (1), 173-183.

Marble, F. E. \& CANDEL, S. M. 1979 An analytical study of the non-steady behavior of large combustors. Symp. (Intl) Combust. 17 (1), 761-769.

Markstein, G. H. 1953 Instability phenomena in combustion waves. Proc. Combust. Inst. 4, 44-59. 
Markstein, G. H. 1964 Non-steady Flame Propagation. Pergamon.

Markstein, G. H. 1970 Flames as amplifiers of fluid mechanical disturbances. In Proceedings of the 6th National Congress for Applied Mechanics, Cambridge, MA, pp. 11-33.

Markstein, G. H. \& SQuire, W. 1955 On the stability of a plane flame front in oscillating flow. J. Acoust. Soc. Am. 27 (3), 416-424.

Matalon, M. \& Matkowsky, B. J. 1982 Flames as gasdynamic discontinuities. J. Fluid Mech. 124, 239-259.

Matkowsky, B. J. \& Sivashinsky, G. I. 1979 An asymptotic derivation of two models in flame theory associated with the constant density approximation. SIAM J. Appl. Maths 37 (3), 686-699.

Michelson, D. M. \& Sivashinsky, G. I. 1977 Nonlinear analysis of hydrodynamic instability in laminar flames - II. Numerical experiments. Acta Astron. 4, 1207-1221.

Noiray, N., Durox, D., Schuller, T. \& Candel, S. 2008 A unified framework for nonlinear combustion instability analysis based on the flame describing function. J. Fluid Mech. 615, $139-167$.

OrszaG, S. A. 1971 On the elimination of aliasing in finite-difference schemes by filtering highwavenumber components. J. Atmos. Sci. 28, 1074.

Pelcé, P. \& Clavin, P. 1982 Influence of hydrodynamics and diffusion upon the stability limits of laminar premixed flames. J. Fluid Mech. 124, 219-237.

Pelcé, P. \& Rochwerger, D. 1992 Vibratory instability of cellular flames propagating in tubes. J. Fluid Mech. 239, 293-307.

PirauX, J. \& LOMBARD, B. 2001 A new interface method for hyperbolic problems with discontinuous coefficients: one-dimensional acoustic example. J. Comput. Phys. 168 (1), 227-248.

Poinsot, T., Trouvé, A., Veynante, D., Candel, S. \& Esposito, E. 1987 Vortex-driven acoustically coupled combustion instabilities. J. Fluid Mech. 177, 265-292.

Preetham, Santosh, H. \& Lieuwen, T. 2008 Dynamics of laminar premixed flames forced by harmonic velocity disturbances. J. Propul. Power 24 (6), 1390-1402.

RastigejeV, Y. \& MAtAlon, M. 2006 Nonlinear evolution of hydrodynamically unstable premixed flames. J. Fluid Mech. 554, 371-392.

Raushenbakh, B. V. 1961 Vibrational Combustion. Fizmatgiz.

RAYLEIGH, LORD 1878 The Theory of Sound, vol. 2. Macmillan.

Schadow, K. C. \& Gutmark, E. 1992 Combustion instability related to vortex shedding in dump combustors and their passive control. Prog. Energy Combust. Sci. 18, 117-132.

Schuller, T., Durox, D. \& CANdel, S. 2003 A unified model for the prediction of laminar flame transfer functions. Combust. Flame 134 (1-2), 21-34.

Searby, G. 1992 Acoustic instability in premixed flames. Combust. Sci. Technol. 81, 221-231.

Searby, G. \& Rochwerger, D. 1991 A parametric acoustic instability in premixed flames. J. Fluid Mech. 231, 529-543.

Searby, G. \& Truffaut, J. M. 2001 Experimental studies of laminar flame instabilities. In Coherent Structures in Complex Systems (ed. D. Reguera, L. L. Bonilla \& J. M. Rubi), pp. 159-181. Springer.

Searby, G., Truffaut, J. M. \& Joulin, G. 2001 Comparison of experiments and a nonlinear model equation for spatially developing flame instability. Phys. Fluids 13 (11), 3270-3276.

Shin, D.-H. \& Lieuwen, T. 2013 Flame wrinkle destruction processes in harmonically forced, turbulent premixed flames. J. Fluid Mech. 721, 484-513.

SIVASHINSKY, G. I. 1977 Nonlinear analysis of hydrodynamic instability in laminar flames - I. Derivation of basic equations. Acta Astron. 4, 1177-1206.

Steinberg, A. M., Boxx, I., Stöhr, M., Carter, C. D. \& Meier, W. 2010 Flow-flame interactions causing acoustically coupled heat release fluctuations in a thermo-acoustically unstable gas turbine model combustor. Combust. Flame 157 (12), 2250-2266.

Trefethen, L. N. 2000 Spectral Methods in MATLAB. SIAM.

VAYnBlat, D. \& MAtAlon, M. 2000a Stability of pole solutions for planar propagating flames: I. Exact eigenvalues and eigenfunctions. SIAM J. Appl. Maths 60 (2), 679-702. 
Vaynblat, D. \& Matalon, M. $2000 b$ Stability of pole solutions for planar propagating flames: II. Properties of eigenvalues/eigenfunctions and implications to stability. SIAM J. Appl. Maths 60 (2), 703-728.

Williams, F. A. 1985 Combustion Theory. Benjamin Cummings.

WU, X. \& LAW, C. K. 2009 Flame-acoustic resonance initiated by vortical disturbances. J. Fluid Mech. 634, 321-357.

WU, X. \& MoIN, P. 2010 Large-activation-energy theory for premixed combustion under the influence of enthalpy fluctuations. J. Fluid Mech. 655, 3-37.

Wu, X., Wang, M., Moin, P. \& Peters, N. 2003 Combustion instability due to the nonlinear interaction between sound and flame. J. Fluid Mech. 497, $23-53$.

YANG, V. \& CULICK, F. E. C. 1986 Analysis of low frequency combustion instabilities in a laboratory ramjet combustor. Combust. Sci. Technol. 45 (1-2), 1-25.

Yu, K. H., Trouvé, A. \& DAILY, J. W. 1991 Low-frequency pressure oscillations in a model ramjet combustor. J. Fluid Mech. 232, 47-72.

Zhang, C. \& LeVeque, R. J. 1997 The immersed interface method for acoustic wave equations with discontinuous coefficients. Wave Motion 25 (3), 237-263. 